\title{
RskA Is a Dual Function Activator-Inhibitor That Controls SigK Activity Across Distinct Bacterial Genera
}

Frédéric J. Veyrier ${ }^{1,2 *}$, Cecilia Nieves ${ }^{1,2}$, Louise H. Lefrancois ${ }^{2,3}$, Hana Trigui ${ }^{1,2}$, Antony T. Vincent ${ }^{1,2}$ and Marcel A. Behr ${ }^{2,3 *}$

${ }^{1}$ Bacterial Symbionts Evolution, Centre Armand-Frappier Santé Biotechnologie, Institut National de la Recherche Scientifique, Université du Québec, Laval, QC, Canada, ${ }^{2}$ McGill International TB Centre, Montreal, QC, Canada,

${ }^{3}$ Department of Medicine, McGill University, Montreal, QC, Canada

\section{OPEN ACCESS}

Edited by:

Riccardo Manganelli,

University of Padua, Italy

Reviewed by:

Daria Bottai,

University of Pisa, Italy

Pallavi Ghosh,

The State University of New York,

United States

*Correspondence:

Frédéric J. Veyrier

frederic.veyrier@inrs.ca

Marcel A. Behr

marcel.behr@mcgill.ca

Specialty section:

This article was submitted to Microbial Physiology and Metabolism,

a section of the journa

Frontiers in Microbiology

Received: 01 May 2020

Accepted: 14 August 2020

Published: 09 September 2020

Citation:

Veyrier FJ, Nieves $C$, Lefrancois $L H$, Trigui $H$, Vincent $A T$ and Behr MA (2020) RskA Is a Dual

Function Activator-Inhibitor That Controls SigK Activity Across Distinct

Bacterial Genera.

Front. Microbiol. 11:558166. doi: 10.3389/fmicb.2020.558166
It has been previously shown that RskA, the anti-Sigma factor $\mathrm{K}$ of Mycobacterium tuberculosis, inhibits SigK and that mutations in RskA promote high expression of the SigK regulon. The latter observation led us to hypothesize that RskA mutations lead to loss of the anti-Sigma factor function. In this report, we used natural and artificial mutations in RskA to determine the basis of the SigK-RskA partnership. Consistent with predictions, the N-terminal cytoplasmic portion of RskA was sufficient on its own to inhibit SigK. Unexpectedly, RskA also served as an activator of SigK. This activation depended on the same $\mathrm{N}$-terminal region and was enhanced by the membraneextracellular portion of RskA. Based on this, we engineered similar truncations in a Gram-negative bacterium, namely Yersinia enterocolitica. Again, we observed that, with specific alterations of RskA, we were able to enhance SigK activity. Together these results support an alternative mechanism of anti-Sigma factor function, that we could term modulator (activator-inhibitor) in both Actinobacteria and Gram-negative bacteria, suggesting that Sigma factor activation by anti-Sigma factors could be under-recognized.

Keywords: M. tuberculosis, M. bovis, M. orygis, Yersinia, Sigma factor K, anti-Sigma factor, RskA

\section{INTRODUCTION}

The Mycobacterium tuberculosis complex (MTC) is a group of genetically similar subspecies responsible for tuberculosis in mammalian hosts (Riojas et al., 2018; Lipworth et al., 2019). Since the divergence of the MTC from the smooth tubercle bacilli (Supply et al., 2013), there is no evidence of gene acquisition by MTC members, and as such, the genomic architecture of MTC organisms is marked by reductive evolution (Mostowy and Behr, 2005). Despite gene deletion and accumulation of loss-of-function mutations, MTC members with more derivative genomes, such as M. bovis, are fully capable of productive infection and transmissible disease in a large number of mammalian hosts. Indeed, M. bovis has the broadest host-range of any MTC member (Morris et al., 1994; O'Reilly and Daborn, 1995): deer, badgers, and opossums have been reported as maintenance hosts (Brites et al., 2018), as well as infection in humans (Scott et al., 2016; Torres-Gonzalez et al., 2016). 
It has been hypothesized that altered gene expression must underlie the capacity of organisms like $M$. bovis to adapt to new host niches, possibly through mutations in negative regulators (Garnier et al., 2003). Consistent with such a model, we previously documented that genes regulated by Sigma factor $\mathrm{K}$ (SigK) are constitutively expressed in $M$. bovis due to mutations in the putative anti-Sigma factor $\mathrm{K}$, known as the Regulator of SigK (RskA) (Said-Salim et al., 2006). This was also suspected to be the case for $M$. orygis (formerly known as the Oryx bacillus) (Supply et al., 2013). We have shown that SigK controls the expression of eleven genes, including mpt70 and mpt83, coding for homologous antigenic proteins MPT70 and MPT83 (Said-Salim et al., 2006), respectively. In addition, it has been reported that the expression of $k a t G$ is influenced by SigK (Sklar et al., 2010).

Sigma factors initiate transcription of targeted genes by directing RNA polymerase to specific gene promoters. Based on phylogenetic relatedness, the $\sigma 70$ family has been divided into several groups (Gruber and Gross, 2003), with those controlling some aspect of the cell surface or transport called ExtraCytoplasmic Function (ECF) Sigma factors (Lonetto et al., 1994). In general, an ECF is co-transcribed with its cognate antiSigma factor, which serves as a negative regulator by preventing the interaction between the ECF and the RNA polymerase until the appearance of a specific signal that allows its release (Helmann, 2002). M. tuberculosis has $13 \sigma$ factors, of which ten correspond to ECF $\sigma$ factors (Manganelli, 2014), SigK being one of them.

The anti-ECF Sigma factor is often a membrane protein that transmits signals across the membrane to the cytoplasmic Sigma factor. Direct sensing of specific stimuli can either be done by the ECF, or by alternative proteins working in concert with the ECF. Several signaling mechanisms for activation of ECF Sigma factors have been described. An example is the FecA/TonB/FecR/FecI model of Escherichia coli, in which FecA initiates the signaling cascade by binding environmental metals which induces structural changes in FecA. With its partner TonB, FecA can interact with the anti-Sigma factor FecR, leading to the release of an active Sigma factor FecI that binds the RNA polymerase (Brooks and Buchanan, 2007). Another example is the SigE model of E. coli. SigE activation occurs upon proteolysis of the anti-Sigma factor RseB/A at the membrane by DegS and RseP proteases. This event releases the part of anti-Sigma factor RseA coupled to the SigE into the cytoplasm, whereupon a cytoplasmic protease cleaves SigE from RseA to initiate transcription (Brooks and Buchanan, 2007). Finally, in the SigB/SigF model of Bacillus subtilis, a "partner-switching" mechanism has been invoked, involving the activity of kinases (Igoshin et al., 2007).

Consistent with other regulators of ECF, $r s k A$ is co-transcribed with sigK. RskA is a predicted transmembrane protein, whose cytoplasmic N-terminal portion directly interacts with SigK (Said-Salim et al., 2006), and has been shown to inhibit the activity of this Sigma factor in different mycobacterial species (Veyrier et al., 2008). In M. tuberculosis, RskA is regulated by sequential proteolysis via RIP1 at cytoplasmic level (Rv2869c) (Makinoshima and Glickman, 2005; Sklar et al., 2010), while the protease responsible for the first cleavage outside the bacteria remains unknown. In addition, it has been shown that the oxidative state of two cysteines (133 and 183) in SigK influences its interaction with RskA and, therefore the signaling (Malkhed et al., 2011; Shukla et al., 2014). Under oxidizing condition, the disulfide bond formed between them stabilizes the SigKRskA complex, whereas, in the presence of a reducing agent, SigK is released (Shukla et al., 2014). However, while RskA has been mutated independently twice in MTC members - M. bovis (G107D, G184E) and M. orygis (X233S) (van Ingen et al., 2014) (Supplementary Figure S1A), in neither case did the mutations affect the cytosolic portion of RskA, where the direct interaction with SigK occurs. It should be noted that the SNPs in the DNA that produce these amino acid changes are the only differences between the sigK-rskA locus from M. tuberculosis, M. bovis and $M$. orygis (i.e., SigK is identical between the three subspecies). While cytosolic RskA mutations might result in an impaired SigK inhibition through defective interaction, the molecular basis by which these extracytosolic mutations could change the expression of the regulon remains unclear (Said-Salim et al., 2006).

In this study, we used natural and artificial mutations in rskA (Supplementary Figure S1B) to improve our knowledge of the molecular mechanism of SigK regulation, revealing an unexpected activating role of RskA. Our findings indicate that RskA is a dual function activator-inhibitor, whereby extracytosolic RskA mutations cause increased SigK activity. In addition, we were able to reproduce these results using a SigK-RskA pair from the Gram-negative bacterium, Yersinia enterocolitica suggesting that this dual function may be widespread in the SigK family.

\section{RESULTS}

\section{Dominant Effect of Naturally Mutated Versions of RskA}

We made a first observation that challenged the simple model of RskA as a pure inhibitor of SigK. Previously, we observed that wild-type RskA from $M$. tuberculosis partially suppressed the expression of the SigK regulon in M. bovis BCG Russia, but only when $r s k A$ was expressed under control of the highly active $h s p 60$ promoter (Said-Salim et al., 2006). Surprisingly, using the same construct, we were unable to suppress SigK activity in M. orygis or virulent $M$. bovis (data not shown). Under the simple inhibition model, it was expected that the absence of a functional RskA would liberate SigK to direct transcription of SigK-regulated genes, so the introduction of wild-type RskA would restore this defect. The difficulties encountered in these complementation studies stimulated an alternative hypothesis, in which mutated versions of RskA (G107D, G184E, or X233S) may be dominant over RskA tuberculosis.

To test this possibility, we generated a plasmid harboring RskA variants along with luciferase fused to the promoter of mpt70 (mpt70p) (Figure 1A). To approximate normal transcriptional conditions, the plasmid (pMV306::hyg) was integrated into the chromosome and the genes were under the control of a putative promoter located just before sigK, 
A

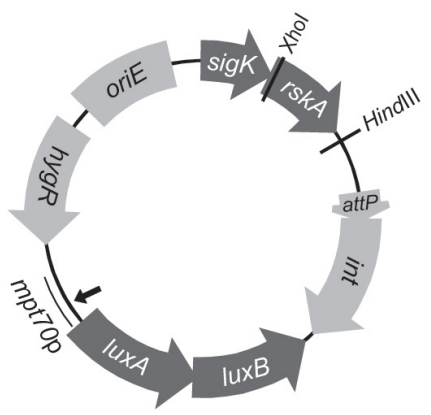

B

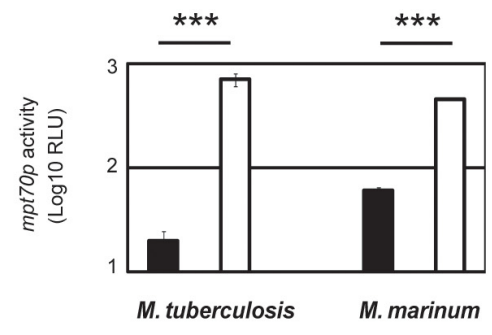

C

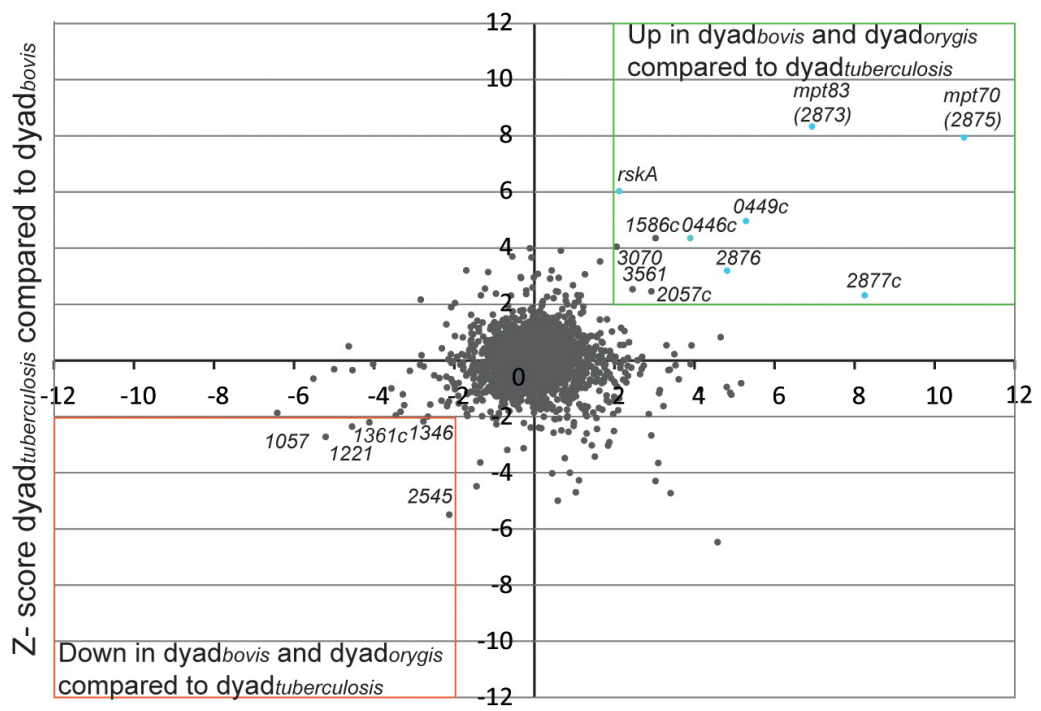

Z- score dyadtuberculosis compared to dyadbovis

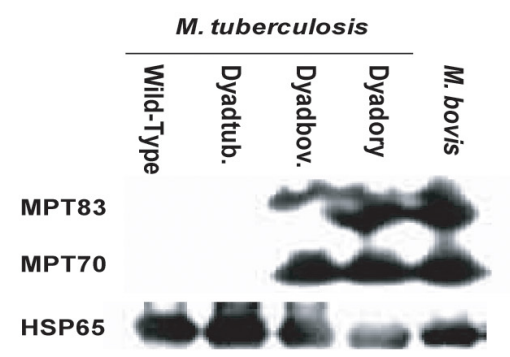

FIGURE 1 | Dominant effect of mutated RskA on wild-type RskA. (A) Schematic representation of the plasmid backbone used in this study. (B) Luciferase expression of $M$. tuberculosis (H37Ra) and M. marinum harboring pMV::DyadRV-Lx70 (black) or pMV::Dyadbovis-Lx70 (white) which expressed, respectively, the sigK-rskA dyad from M. tuberculosis and M. bovis. Error bars represent standard deviations. Mutant forms of $r$ skA result in over-expression of the SigK regulated genes, even in the presence of the wild-type $r s k A$. Each bar represents the mean measurement for three independent clones ( $\left.{ }^{\star \star \star} p<0.001\right)$. This representative data has been replicated at least twice. (C) Comparison of the activity of dyad bovis and dyad orygis $_{\text {with dayd }}$ tuberculosis $_{\text {assessed by microarray. }}$-scores for M. tuberculosis H37Rv dayd tuberculosis (with pMV-Hyg::Dyad) compared with Dyad bovis. (pMV::Dyadbovis) ( $y$ axis, representing average of two arrays) have been

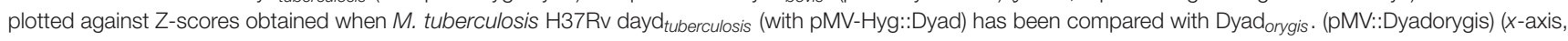
representing the average of two arrays). Genes whose expression is up-regulated in both cases are for the majority part of the sigK or $m p t 70$ locus (light blue). (D) Expression of MPT70, MPT83 and HSP65 (Loading control) measured by Western blot for M. tuberculosis H37Rv in native state (lane 1), M. tuberculosis after introduction of different sigK-rskA dyads (lanes 2-4) and M. bovis (lane 5). Dyadtub. indicates pMV-Hyg::Dyad, Dyadbov. indicates pMV::Dyadbovis and Dyadory indicates pMV::DyadOryx. This representative data has been replicated twice.

referred to as sigKp (Veyrier et al., 2008). The SigK activity reporter system was constituted by the $\operatorname{lu} x A B$ genes from Vibrio harveyi controlled by mpt70p from M. tuberculosis, which we previously demonstrated is regulated only by SigK in vitro (Veyrier et al., 2008). These constructs were introduced in M. tuberculosis wild-type and M. marinum. Rather than including rskA alone, we used gene dyads (sigK-rskA) to assure that the ratio SigK/RskA is not modified. The luminescence produced by these bacteria is presented in Figure 1B. In M. tuberculosis, introduction of dyad bovis $_{\text {strongly increased } m p t 70 \text { promoter }}$ activity in the presence of wild-type RskA. The same results were observed in M. marinum which also possesses an inducible SigK regulon (Veyrier et al., 2008). To confirm that these results were not biased due to a problem in the plasmid, the M. tuberculosis genomic promoter response was directly evaluated using microarrays (Figure 1C) and indirectly by measuring the expression of MPT70 and MPT83 by immunoblot (Figure 1D). Our results show that the introduction of the dyad $_{\text {tuberculosis }}$ into $M$. tuberculosis did not affect the expression of MPT70 and MPT83. In contrast, the introduction of both dyad $_{\text {bovis }}$ and dyad $\mathrm{d}_{\text {orygis }}$ increased production of these proteins to a similar level than that observed in M. bovis. Taken together, 

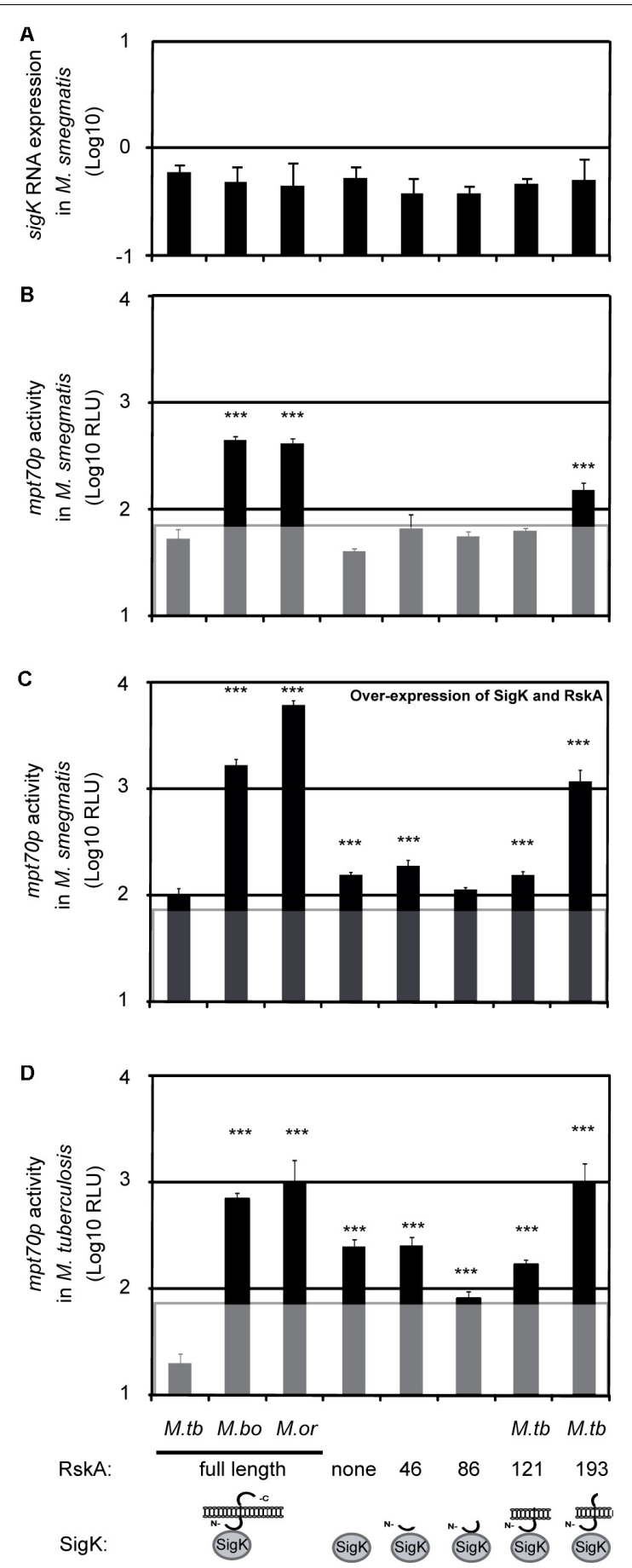

FIGURE 2 | The anti-Sigma factor $K$ (RskA) from $M$. tuberculosis is an activator and an inhibitor of SigK. (A) Relative expression of sigK, measured by RT-qPCR normalized with sigA, in $M$. smegmatis harboring the indicated constructs. Each bar represents the mean measurement for three independent clones. (B-D) Effects of RskA truncation or mutation on SigK activity, measured using luciferase under control of the promoter of mpt70. (B) Experiment conducted in the absence of a wild-type RskA (M. smegmatis). (C) Experiment conducted in the absence of a wild-type RskA (M. smegmatis) with over-expression of SigK and RskA and (D) Experiment conducted in the presence of a wild-type RskA

(Continued)
FIGURE 2 | Continued

(M. tuberculosis H37Ra). Error bars represent standard deviations for measurement of three independent clones. The background noise (line) was set at 1.8, based on replicate measurements of $M$. smegmatis expressing the luciferase under the promoter of $m p t 70$ but without sigK and rskA (data not shown). M.tb indicates $M$. tuberculosis, $M$. bo indicates $M$. bovis and M.or indicates $M$. orygis. Depending on the RskA forms, variable degrees of SigK modulation were observed. This representative data has been replicated twice $\left({ }^{* \star *} p<0.001\right.$ compared with dyadtuberculosis $)$.

these findings support the hypothesis that the mutated versions are dominant on the wild-type RskA.

\section{M. bovis and M. orygis RskA Variants Activate SigK}

To explain the results seen with $\mathrm{RskA}_{\text {bovis }}$ and $\mathrm{RskA}_{\text {orygis }}$, we hypothesized that (1) RskA can act as an activator of SigK, and (2) mutated versions of RskA from M. bovis and M. orygis are not merely non-functional - rather, these mutations result in SigK remaining in an activated state. To formally test the effect of these different natural mutants of RskA in a standardized and easy-to-use assay, we chose a mycobacterial species that lacks the sigK-rskA dyad, namely $M$. smegmatis. Into this organism, we introduced the plasmids containing both the sigK-rskA dyad regulatory core and a SigK-regulated reporter system (see Figure 1A). Moreover, we anticipated that this heterologous assay would be feasible because the SigK-binding sites are highly conserved between members of Actinobacteria, and because orthologues of SigK from different species can recognize the site in the M. tuberculosis mpt70 promoter (Veyrier et al., 2008).

To validate the assay, we first tested it for already-documented effects of RskA mutations, by introducing plasmids coding for SigK along with different versions of RskA (from M. tuberculosis, $M$. bovis, or $M$. orygis). As expected, the dyad $\mathrm{d}_{\text {bovis }}$ and the dyad $_{\text {orygis }}$ resulted in $\sim 6$-times more luciferase expression than

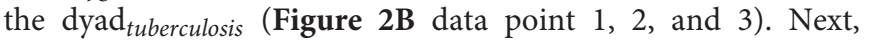
we measured the activity of SigK alone by removing RskA from the plasmid previously described (pMV::dyadbovis-Lx70) and measuring luciferase activity in $M$. smegmatis (Figure 2B, data point 4). In the presence of full-length RskA bovis (data point 2) or RskA $A_{\text {orygis }}$ (data point 3), we observed RLU superior than that observed when RskA was absent (i.e., SigK alone, data point 4). Therefore, in addition to the inhibitor function of RskA (documented previously, Said-Salim et al., 2006), it can be an activator of SigK activity. As a control, we have measured sigK RNA expression using RT-qPCR in M. smegmatis harboring the different plasmids to test for constructionmediated changes in sigK expression which could influence SigK activity. Control measurements showed no significant differences in sigK expression between constructs (Figure 2A). To test whether removing the RskA $\mathrm{A}_{\text {bovis }}$ or Rsk $\mathrm{A}_{\text {orygis }}$ had similar effects in $M$. tuberculosis, we introduced the same constructs into the H37Ra strain of $M$. tuberculosis. As shown in Figure 2D, the presence of $\mathrm{RskA}_{\text {bovis }}$ (data point 2) or RskA orygis (data point 3) resulted in superior activity than the one observed when SigK is alone (data point 4). Of note, in M. smegmatis, the plasmid 
A
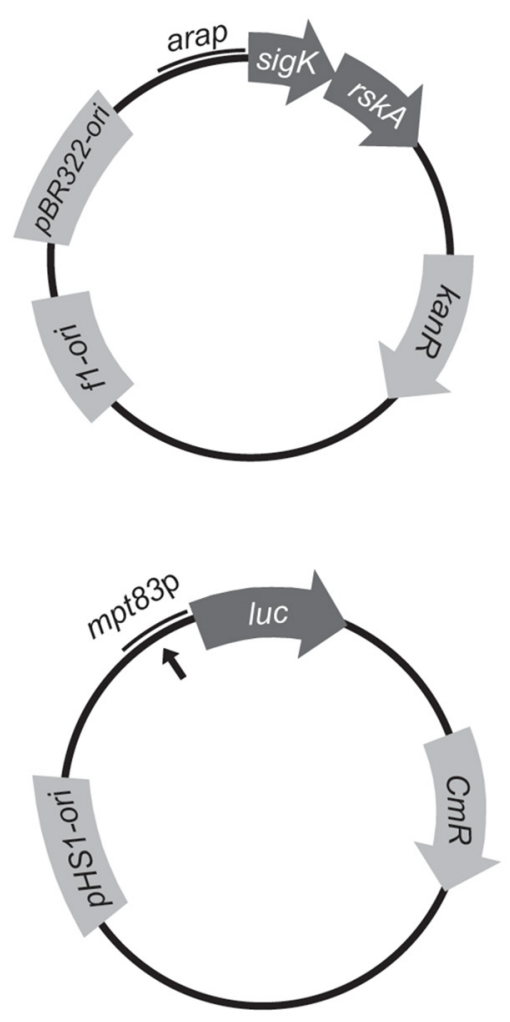

B

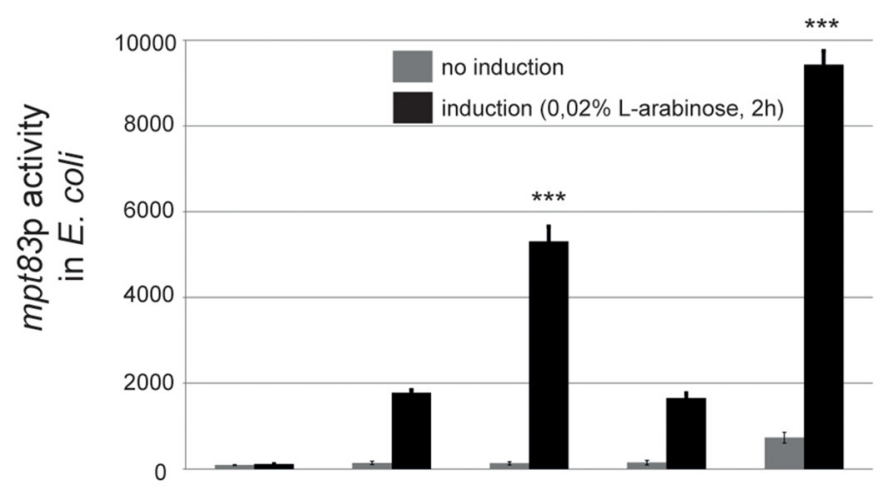

C

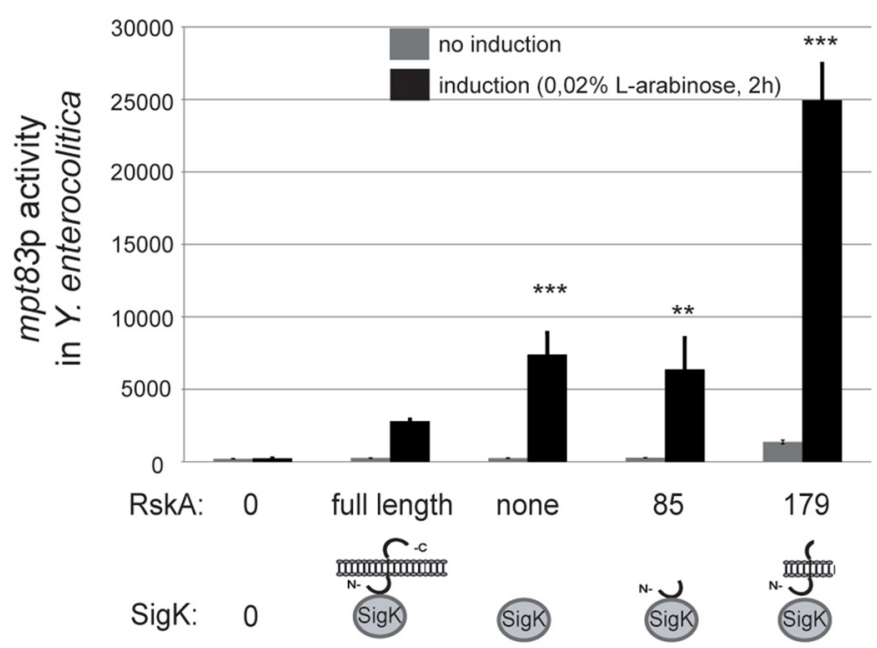

FIGURE 3 | (A) The anti-Sigma factor K (RskA) from Y. enterocolitica is an activator and an inhibitor of SigK. (A) Schematic representation of the plasmid backbones used in this study. Bold black arrow represents predicted SigK binding site. Truncated versions of RskA from Y. enterocolitica and its effect on SigK activity measured by luciferase expression under control of mpt83 promoter in E. coli (B), and Y. enterocolitica (C). Error bars represent standard deviations of three independent experiments. Non-induction and induction with L-arabinose are shown in both cases $\left({ }^{\star *} p<0.01,{ }^{\star \star *} p<0.001\right.$ compared with SigK and full-length RskA).

versions of the dyads are under control of sigKp, which is not SigK regulated, enabling us to quantify the expression of our construct reliably. Besides, we reproduced this effect by changing sigKp by the overexpressing promoter hsp60p (Figure 2C). However, in M. tuberculosis, the genomic version of the gene is autoregulated via Rv0449cp (Charlet et al., 2005; Said-Salim et al., 2006; Veyrier et al., 2008), therefore we have not quantified sigK expression by RT-qPCR.

\section{Artificial Truncation Can Also Block RskA Into Its SigK-Activating Conformation}

In previous studies with FecI-FecR, a similar effect of FecIactivation by FecR was observed using artificial truncation of the anti-Sigma factor (Ochs et al., 1995, 1996). To further explore the Sigma/anti-Sigma partnership, beyond the natural mutation, we constructed different versions of the plasmid coding for SigK along with truncated versions of RskA. Schematic representations of the different RskA truncations are presented at the bottom of Figure 2 (data points 5-8) and in Supplementary Figure S1. Again, sigK expression by RT-qPCR was similar across constructs, enabling us to infer that any differences observed were due to the RskA variants (Figure 2A). Interestingly, we could observe that the removal of a specific fragment of the C-terminal part of $\mathrm{RskA}_{\text {tuberculosis }}$ (194 to 232, data point 8) led to the same phenotype observed with RskA $\mathrm{A}_{\text {bovis }}$ or RskA $\mathrm{A}_{\text {orygis }}$ (data points 2 and 3) whereas further truncation (122 to 232, data point 7 or 87 to 232 , data point 6) did not. This suggests that the extra cytoplasmic tail (194 to 232) is controlling the dual activity of RskA and removing it has the same effect as mutation (G107D, G184E, or X233S).

\section{Artificial Truncation Can Also Block RskA Into Its SigK-Activating Conformation in Gram-Negative Bacteria}

To explore whether the findings are specific to Mycobacteria, we tested this model in a genetically different bacteria that harbor mpt83-sigK-rskA minimal cassette (Veyrier et al., 2008) namely $Y$. enterocolitica. To prevent confusion, MPT83 and MPT70 are paralogous genes in $M$. tuberculosis that arose from a duplication. We have already shown that MPT83 (with a 


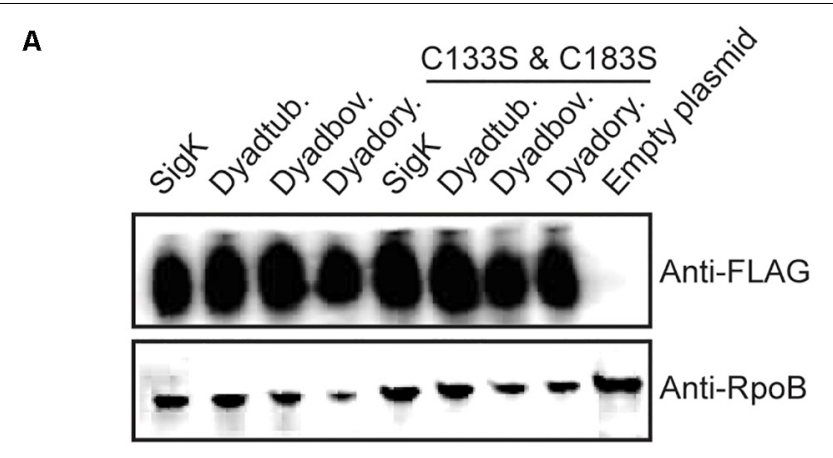

M. smegmatis

B

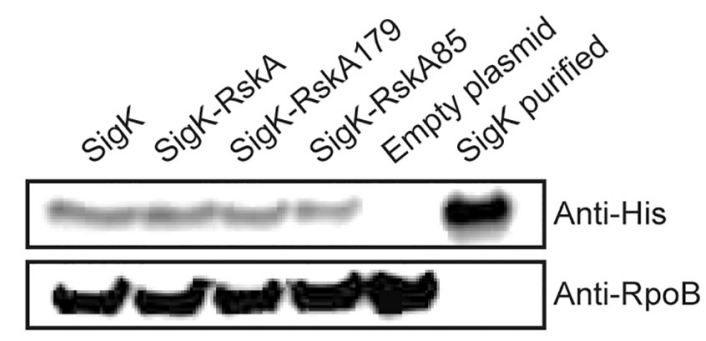

E. coli

FIGURE 4 | SigK activity occurs without degradation of SigK protein. (A) Expression of SigK, with a FLAG-tag linked to the N-terminus, was detected by immunoblot using monoclonal FLAG-tag antibodies in M. smegmatis wild-type, or expressing SigK alone; Dyadtuberculosis, Dyad bovis $_{\text {s. }}$ and Dyadorygis and the SigK-cysteine-less versions. (B) Similarly, expression of SigK with a His-tag linked to the $\mathrm{N}$-terminus, was detected by immunoblot using monoclonal His-tag antibodies in E. coli expressing SigK alone; SigK-RskA; SigK-RskA179 or SigK-RskA85. Purified SigK was used as a positive control and $E$. coli harboring the empty plasmid as a negative control. RpoB was used as a loading control in both cases using an anti-RpoB antibody that react with both species. This representative data has been replicated twice.

lipid anchor) is the ancestral version (Veyrier et al., 2008). Therefore, we called the gene mpt83 (Veyrier et al., 2008) and have already shown that a SigK-binding site is present upstream of the gene. A similar strategy was taken with heterologous expression in E. coli (another proteobacteria but that do not contain SigK nor RskA) and Y. enterocolitica wild-type. We constructed a plasmid (pST76-K) that harbors mpt83p from $Y$. enterocolitica fused to the firefly luciferase (Figure 3A). Different versions of sigK and rskA were cloned into $\mathrm{pBAD}$ and expressed with the arabinose inducible promoter (Figure 3A). We expressed truncated versions of RskA $A_{\text {enterocolitica }}$ (1 to 179) or (1 to 85), which correspond to the same truncations studied for RskA tuberculosis: $_{\text {: }}(1$ to 193 ) and (1 to 86 ), respectively (see the alignment in Supplementary Figure S1). Again, whether expressed in E. coli (Figure 3B) or Y. enterocolitica (Figure 3C), RskA had the same effect on SigK: no activity with the full-length RskA or with 1 to 85 version (inhibition); some activity with SigK alone (low on); full activation with RskA from 1 to 179 (high on). These data suggest that the mechanism of SigK activation by RskA is conserved across these genera.

\section{RskA Does Not Stabilize SigK}

Based on prior studies on SigK activation, we hypothesized that RskA could undergo sequential proteolysis and that a small remaining fragment of RskA would stabilize SigK. Proteolysis of Sigma factors has been demonstrated for SigT in Streptomyces coelicolor (Mao et al., 2013). Therefore, we tested if SigK is stable with the different versions of RskA in both mycobacteria and proteobacteria. To evaluate this possibility, we first added a FLAG-tag at the N-terminal part of SigK $\mathrm{K}_{\text {tuberculosis }}$ and showed that the activity was unaffected by the tag (Supplementary Figure S2A). Next, we measured SigK levels by immunoblot against FLAG-tag in M. smegmatis, revealing that SigK activity, which varies widely according to the RskA, was uncoupled from the amount of SigK detected (Figure 4A). We performed the same in proteobacteria by expressing the different versions of the dyad with a His-tag in the $\mathrm{N}$-terminal part of SigK $\mathrm{K}_{\text {enterocolitica }}$ in E. coli using pET-28a plasmid. Again, the tag did not alter the activity of SigK (low on, on and high on) (Supplementary Figure S2B) and we still did not observe changes in SigK stability correlated with its activity (Figure 4B).

\section{SigK-Cysteines Are Important for Its Activity}

Cysteines (133 and 183) in SigK from M. tuberculosis in their oxidized state have been shown to influence the RskA-SigK interaction, and therefore signaling (Malkhed et al., 2011; Shukla et al., 2014). We tested whether interfering with the interaction would affect activity by replacing these two cysteines with serines. As seen in Figure 5, activation was abrogated for both the Dyad $_{\text {bovis }}$ and Dyad $\mathrm{d}_{\text {orygis }}$ in M. smegmatis (Figure 5A) and in M. tuberculosis $\Delta$ sigK (Supplementary Figure S3). To verify that these SigK versions are all expressed and stable, we used a FLAGtag fusion strategy as explained before and we measured SigK levels by immunoblot in M. smegmatis, revealing that SigK and cysteines-less SigK are expressed similarly (Figure $\mathbf{4 A}$ ).

We performed a similar experiment in E. coli by expressing the different versions of RskA $\mathrm{A}_{\text {enterocolitica }}$, again with SigK where cysteine 181 was replaced by a serine (Figure 5B). Without these cysteines, SigK activity is minimal and no activation by the truncated RskA is observed. This data supports a model where SigK requires the two cysteines to be fully activated by RskA.

\section{Bioinformatic Search for Anti-Sigma Factor Adaptation Toward Cysteines Absence in SigK}

Several studies have grouped Sigma factors in different families (Staron et al., 2009; Pinto and Mascher, 2016). We present in Figure 6 a phylogeny of all the Sigma factors (Figure 6A), showing that SigK $\mathrm{K}_{\text {tuberculosis }}$ is part of the ECF18 and $\mathrm{SigK}_{\text {enterocolitica }}$ is part of the neighboring ECF19 family (Figure 6B). To study these two families in more detail, we searched for SigK in all the representative genomes of the RefSeq database (11,072 sequences). We recovered $4157 \mathrm{SigK}$ sequences 
A SigK-RskA system in mycobacteria

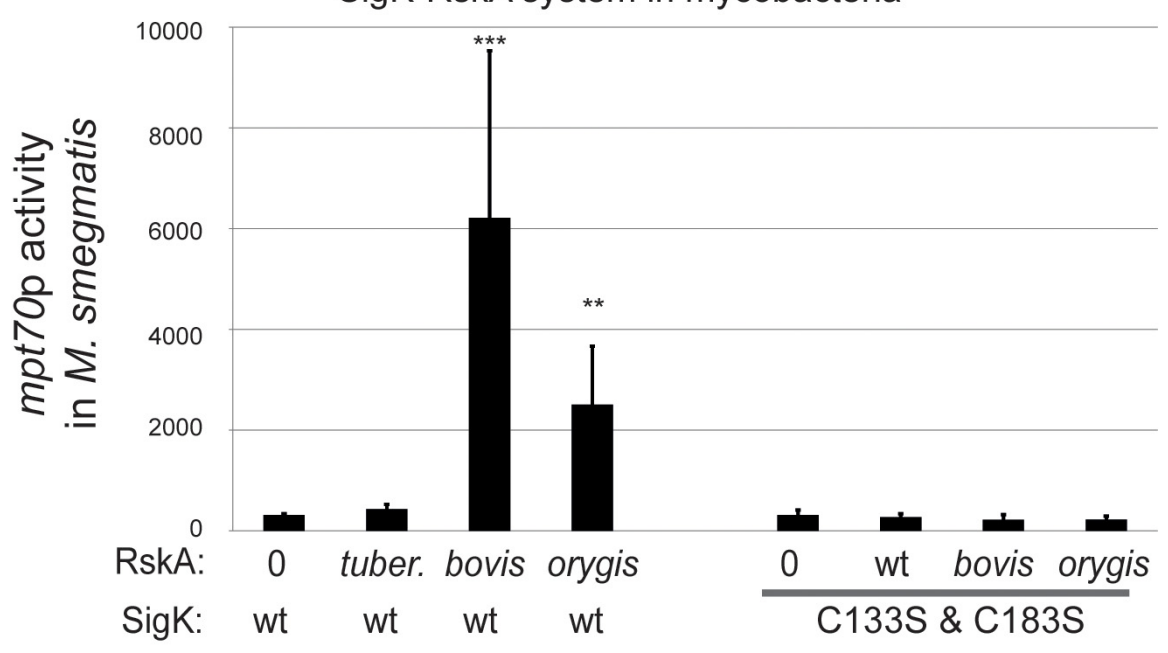

B

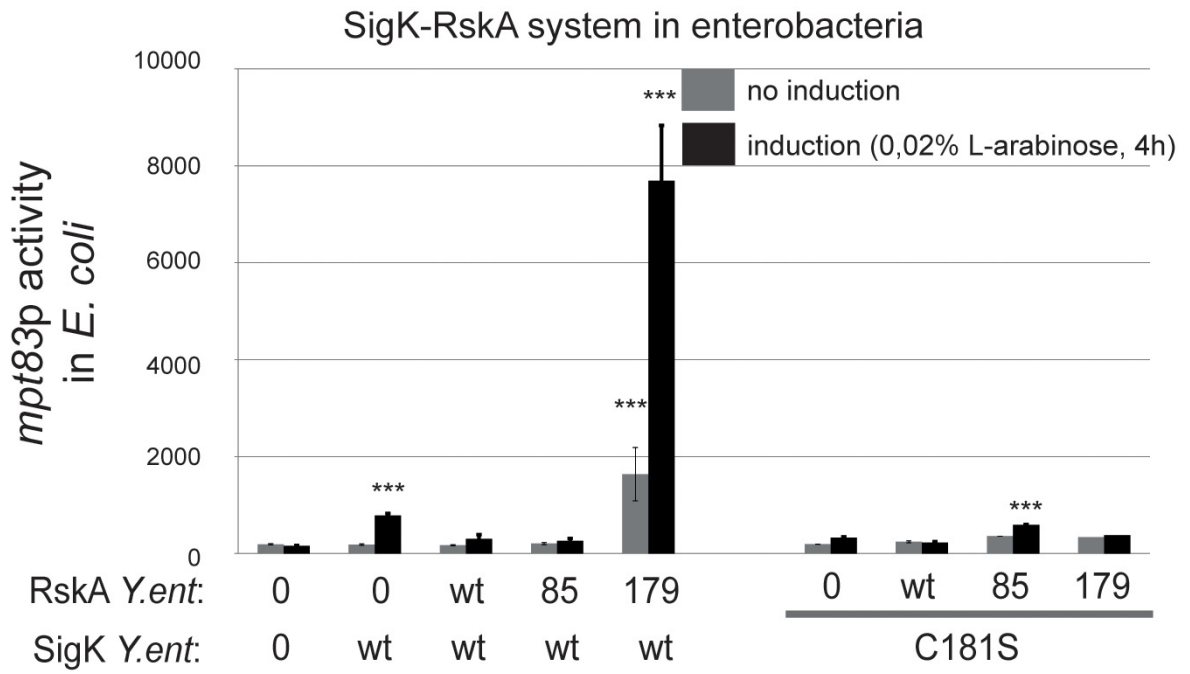

FIGURE 5 | SigK activity requires two conserved cysteines carried by SigK. Effect of cysteines vs. serines on SigK activity were measured by luciferase under the control of mpt70p in M. smegmatis (A) and mpt83p in E. coli (B). Error bars represent standard deviations of three independent experiments with 2 different clones each time. ${ }^{* *} p<0.01,{ }^{* * *} p<0.001$.

distributed across 22 families (Supplementary Table S1). As expected, we found around $35 \%$ of ECF 18 and $35 \%$ of ECF 19 . The other represented families are ECF01 (11\%); ECF34 (8\%); ECF11 (6\%); and ECF70 (3\%). Next, we aligned all the proteins and checked for cysteines 133 and 183 conservation. These cysteines are conserved in $94 \%$ in ECF18 and in 56\% in ECF19, but less than $2 \%$ of other ECF families, suggesting that this may be a specific feature of these two families. To better characterize the partnership between SigK and RskA and the role of these cysteines, we searched for RskA homologous sequences by BLASTP with the requirement that the RskA be adjacent to the identified SigK, considering the polarity of the genes. In the event that no RskA was found, the adjacent gene was still extracted. We next analyzed the obvious differences in RskA between loci that contain SigK (ECF18 and ECF19) with or without the two cysteines (Figure 6C). We first verified the presence of RskA. The absence of RskA was defined by a complete absence of antiSigma factor or an anti-Sigma factor from a different family than RskA. In the group SigK with the 2 cysteines, $98 \%$ of them harbor a RskA whereas this proportion decrease to $85 \%$ in the group of SigK without cysteines (Figure 6C). In repeated instances, we detected species with a truncated RskA (with an arbitrary threshold of $<150 \mathrm{AA}$ ). In the group SigK with the 2 cysteines, $0.3 \%$ of them harbor a truncated RskA; this proportion increased to $9 \%$ in the group of SigK without cysteines (Figure 6C). Overall, $23 \%$ of SigK without the 2 cysteines harbor abnormal RskA (as compared to $2 \%$ in SigK with cysteines) (Figure 6C). This firmly suggests a strong ongoing adaptation of this Sigma factor/antiSigma factor partnership for several species that have members from these two families of ECF18 and ECF19 (as we first detected 

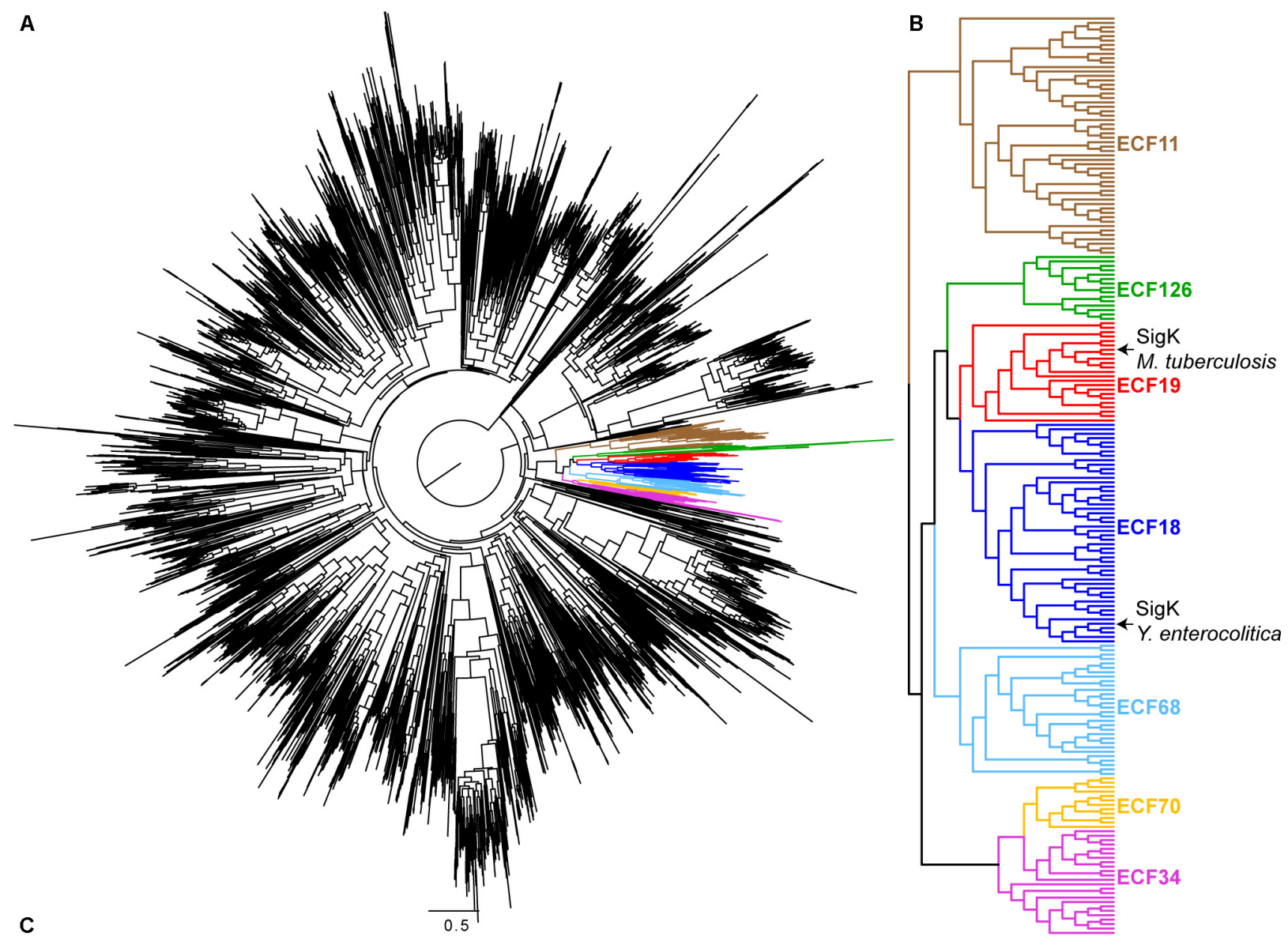

\begin{tabular}{|c|c|c|c|c|c|c|c|c|}
\hline & $\begin{array}{l}\text { Grouped } \\
\text { SigK in } \\
\text { specified } \\
\text { ECF (\%) }\end{array}$ & $\begin{array}{l}\text { SigK with } \\
\text { conserved } \\
\text { cysteins (133, } \\
183)(\%)\end{array}$ & $\begin{array}{l}\text { Sigk with the } \\
\text { conserved } \\
\text { cysteins that } \\
\text { have RskA (\%) }\end{array}$ & $\begin{array}{l}\text { Sigk without } \\
\text { cysteins that } \\
\text { have RskA } \\
(\%)\end{array}$ & $\begin{array}{l}\text { Sigk with the } \\
\text { conserved cysteins } \\
\text { that have truncated } \\
\text { RskA (\%) }\end{array}$ & $\begin{array}{l}\text { Sigk without } \\
\text { cysteins that } \\
\text { have truncated } \\
\text { RskA (\%) }\end{array}$ & $\begin{array}{l}\text { Sigk with the } \\
\text { conserved cysteins } \\
\text { that have abnormal } \\
\text { RskA (\%) }\end{array}$ & $\begin{array}{l}\text { Sigk without } \\
\text { cysteins that } \\
\text { have abnormal } \\
\text { RskA (\%) }\end{array}$ \\
\hline ECF18 & 34,90 & 94,69 & 98,40 & 70,13 & 0,30 & 1,85 & 1,89 & 31,17 \\
\hline ECF19 & 34,69 & 55,76 & 97,26 & 86,68 & 0,38 & 9,58 & 3,11 & 21,63 \\
\hline Total ECF18 and 19 & 69,59 & 75,29 & 97,98 & 84,90 & 0,33 & 8,90 & 2,34 & 22,66 \\
\hline Other ECFs & 30,41 & 1,11 & NA & NA & & & & \\
\hline
\end{tabular}

FIGURE 6 | Species with SigK that naturally evolved without cysteines have adapted RskA (A) Phylogenetic tree of 3717 ECF sequences done as described in the Materials and Methods section. Specific ECF families are colored, as also shown in panel (B). (C) Statistics on the SigK/RskA based on all representative sequences available and found in RefSeq, as described in the section "Materials and Methods".

in $M$. bovis and M. orygis) and particularly for those that do not have conserved these two cysteines.

\section{SigK Without Cysteines, Found in Nocardia spp., Are Functional Without RskA Activation}

When we mutated the cysteines, SigK activity was minimal and RskA activation was not observed. But several species harbor SigK without cysteines. To understand these natural cysteine-less SigK, we again used an evolutionary method, looking for natural mutants, in this case outside of the M. tuberculosis complex. We previously described a cassette consisting of mpt83-sigK-rskA (Veyrier et al., 2008) with a SigK binding site that is conserved even in Gram-negative bacteria (e.g., Yersinia sp.). The genera
Nocardia sp., Rhodococcus sp. and Mycobacterium sp. are part of the same suborder Corynebacterineae. While both Rhodococcus sp. and Nocardia farcinica have a full-length sigK, in N. farcinica the $r s k A$ is truncated (coding for a protein of 81 amino acids) and $m p t 83$ is absent (Figure $7 \mathbf{A}$ ). To test whether the premature stop codon was a general feature of Nocardia sp., we sequenced this region in Nocardia asteroides 42007, N. nova 90960, and $N$. abscessus 91107 . In each of these species, we detected the same stop-codon mutation (and the absence of mpt83). Interestingly, the cysteines are absent in these species (whereas they are conserved in all the other mentioned species harboring a SigK and a non-truncated RskA)

To test the potential consequence of this form of RskA and SigK, we introduced the sigK-rskA dyad from Nocardia asteroides into the plasmid containing $l u x A B$ under the control of $m p t 70 p$ 


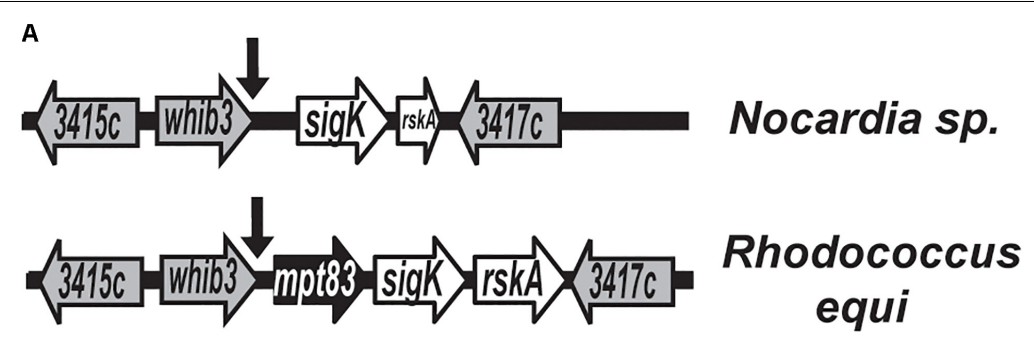

B

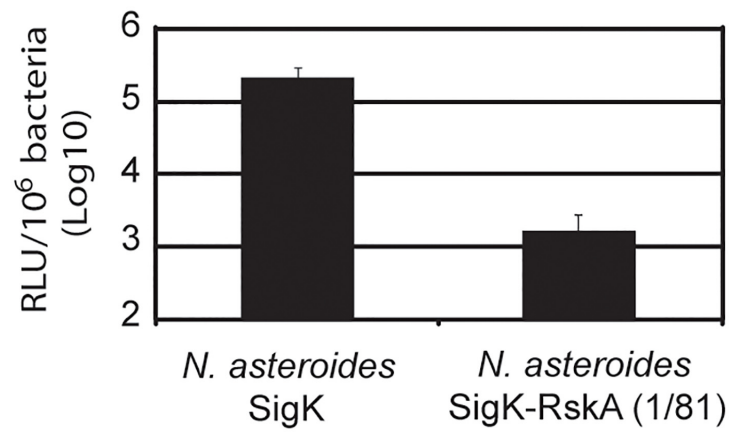

FIGURE 7 | SigK that naturally evolved without cysteine is functional. (A) Schematic representation of sigK locus in Nocardia sp. and Rhodococcus equi. In Nocardia, the rskA gene encodes a truncated protein (1 to 81) and mpt83, a core member of the conserved mpt83-sigK-rskA cassette (Veyrier et al., 2008), has been deleted. The arrows indicate SigK binding sites and genes are named based on their M. tuberculosis H37Rv number. (B) Luciferase expression of M. smegmatis with plasmid containing sigK nocardia or sigK $K_{\text {nocardia }}$ and $r$ sk $A_{\text {nocardia }}$ (1 to 81 ). Error bars represent standard deviations. In the absence of the first 81 amino acids of RskA, there is a strong activation of mpt70p. Each bar represents the mean measurement for three independent clones. This representative data has been replicated at least twice.

(Supplementary Figure S1). As a control, we engineered a RskAless plasmid, by introducing a stop codon in the rskA gene after sigK stop codon. After electroporation into M. smegmatis, we observed that removing the remaining part of RskA results in a $\sim 2 \log$ increased in luciferase activity compared to SigK alone (Figure 7B). These findings indicate that SigK alone is highly active and the presence of the first 81 amino acids of RskA $A_{\text {asteroides }}$ inhibited SigK $\mathrm{K}_{\text {asteroides }}$. Of note, we have previously shown that amino acids 1 to 92 from RskA are sufficient to bind SigK (Said-Salim et al., 2006) and have demonstrated that amino acids 1 to 81 of RskA inhibit SigK (Figures 2, 3). These data support a model in which RskA, like any other antiSigma factors, acts as an inhibitor of its cognate Sigma factor, through its N-terminal portion. In the specific case of Nocardia sp., in the absence of RskA we observed a strong induction of $m p t 70 p$, to a much greater degree than was observed in the case of SigK $\mathrm{K}_{\text {tuberculosis }}$ alone and similar to what was observed with dyad $_{\text {bovis }}$ or dyad $\mathrm{d}_{\text {orygis }}$. This suggests that $\operatorname{SigK}_{\text {asteroides }}$ is able to strongly act on $m p t 70 p$ promoter without the need for a RskA-mediated switching event. A unique aspect of this particular Sigma factor is the absence of the two cysteines which are conserved in other SigK sequences from ECF18 and ECF19. It is important to note that with recent sequencing efforts and increased Nocardia genome sequences available for comparison, certain strain of Nocardia with the archetype of SigK and RskA can be observed (Supplementary Table S1): for example, Nocardia sp. 348MFTsu5.1 harbors a full-length RskA (H290_RS0112295) and a SigK with the two cysteines (H290_RS0112290), indicating again that the these two cysteines in SigK are found concomitantly with the ancestral fulllength RskA.

\section{DISCUSSION}

The detailed study of Sigma factor activation offers insights into the processes used by bacteria to respond to the stresses they encounter during their lifecycle. In this study, we have explored natural and engineered mutations in RskA (the anti-Sigma factor of SigK) to investigate the signal transduction process of the SigK regulon in Actinobacteria, and also Gram-negative bacteria. In Mycobacteria, using a number of different approaches, our investigations demonstrate that increased SigK activity on the SigK-regulated promoter does not happen simply because of lossof-function mutations in the respective forms of RskA in M. bovis and $M$. orygis. Rather, these two independently mutated forms of RskA, whether introduced into M. smegmatis or M. tuberculosis, actively turn on SigK, even in the presence of wild-type RskA. We also demonstrated that the same effect could be obtained by removing a part of the C-terminal part of RskA in both Mycobacteria and Enterobacterales. Based on our results, we infer that SigK has at least three degrees of activity: (i) "Off" when 
inhibited by RskA, (ii) "Low-On" when alone, and (iii) "HighOn" when activated by RskA. The first two states are generally used to identify a protein as an anti-Sigma factor, unlike the third condition (activation) which is rarely tested. One exception is the anti-Sigma factor FecR which has been shown by different groups to activate the cytoplasmic Sigma factor FecI in different bacteria (Ochs et al., 1995; Llamas et al., 2009; Llamas et al., 2009). Interestingly, the description of FecI activation by FecR would have been impossible without mutagenic studies of FecR (Ochs et al., 1995, 1996), in the same way that the activating properties of RskA would not have been suspected if not for the natural mutants in $M$. bovis and $M$. orygis. Importantly, there are clear differences between the FecR model and our data on RskA. The key distinction is that the FecI-modulating domain of FecR (Nterm 1 to 85 ) is an activator when separated from the rest of the protein (Stiefel et al., 2001). In RskA, the equivalent domain of RskA is an inhibitor on its own. Based on this, we predict that the activating function of other anti-Sigma factors could be underappreciated.

As the signal that activates the signaling cascade is still unknown (we have not found any condition that is able to activate $m p t 70$ p, except contact with macrophage, Veyrier et al., 2008), we were unable to test the precise events that initiate the cascade. In M. tuberculosis, it has been shown that RskA is hydrolyzed by an intramembrane hydrolase Rip1(Rv2869c) that belongs to site-2-protease (S2P) (Sklar et al., 2010). The S1P protease of RskA in $M$. tuberculosis remains unknown. Interestingly, a recent study showed that some anti-Sigma factors have an auto-cleavage between the periplasmic Gly191 and Thr192 residues (Bastiaansen et al., 2015). These residues are present in RskA and this motif is the one mutated (G193E) in M. bovis. Furthermore, we have shown that the C-terminal part of RskA has an inhibitory effect on RskA activating function. Our findings, along with observations from other organisms, suggest that activation of SigK by RskA could start with a modification of C-terminal portion of RskA. Therefore, a possible hypothesis would be that a sequential proteolysis of RskA could result in a small fragment of RskA that promotes SigK activity, as has been proposed for FecIR and some anti-Sigma factors from Pseudomonas sp. (Otero-Asman et al., 2019), and alteration of the C-terminal part of RskA exacerbates this. Although the mechanism of cascade activation is not understood, our data report on the signal transmission mechanism between RskA and SigK. A recent study determined the crystal structure of SigK in complex with the cytoplasmic domain of RskA and revealed the presence of disulfide bridge involved in the stability of the partnership (Shukla et al., 2014). Our findings support and build upon these results. We have confirmed the importance of these cysteines in signal transduction of SigK in both Mycobacteria and Enterobacterales. One possible mechanism for SigK activation through RskA would be through the protection against proteases of SigK by RskA, as has been demonstrated in Streptomyces coelicolor (Mao et al., 2013). In our model, the inactivated SigK system of $M$. tuberculosis does not appear to occur through SigK instability. Finally, it could also be possible that SigK recognizes a signal through the cysteine bridge and that RskA is modulating this sensing via its interaction with SigK.
Interestingly, upon further characterization of the ECF18 and ECF19, we also shed light on the "recent" ongoing evolution of these family of Sigma factor/anti-Sigma factors in multiple instances. The co-occurrence of the cysteines with full-length RskA suggests a to-be-determined role of these cysteines in the switching On/Off of SigK by full-length RskA. We found that absence of the two cysteines is correlated with changes in the anti-Sigma factor (as compared to the RskA potential archetype). This could be the presence of another anti-Sigma from a different family than RskA but also, in numerous cases, mutations in RskA. For example, we found that the majority of Nocardia spp. have evolved a Sigma-factor without cysteine and a truncated RskA (around 80 amino acids). It is interesting to note that, unlike when we artificially mutated the cysteines in SigK, naturally evolved cysteine-less SigK are highly active suggesting of compensatory amino-acid changes. In addition, the little remnant of RskA, although often annotated as a pseudogene, is still "functional" and serves as an inhibitor of SigK. The species-specific evolution of this family is complexifying the study of the SigK/RskA partnership. In the light of these examples of species-specific evolution (M. bovis, M. orygis, Nocardia spp.) the study of the partnership between SigK and RskA could be complicated and imply some species-specific characteristics. Nevertheless, these species have all lost or altered the part of RskA that is supposed to sense the signal. Again, this may argue for the possibility that SigK would also sense a specific/alternative signal.

In conclusion, our results emphasize a broader and complex function for anti-Sigma factors as modulators (inhibitor/activator) of the Sigma factor, rather than solely acting as inhibitors. Importantly, the nature of the signal which initiates this cascade is still unknown. Nevertheless, this knowledge can serve to engineer differential expression of the SigK regulon in the same bacterial background and generate tools to decipher in detail the transcriptional activation and the function of this regulon. A similar strategy, using natural and engineered anti-Sigma factor mutations, represents an attractive approach to control the activity of specific Sigma factors, which serves to understand the function of their regulons.

\section{MATERIALS AND METHODS}

\section{Bacterial Strains, Culture Conditions, and Genomic DNA}

Mycobacterium orygis 51145 (Mostowy et al., 2005; Said-Salim et al., 2006), M. bovis 68799 (gifts from Louise Thibert), M. tuberculosis $\mathrm{H} 37 \mathrm{Rv}$, M. tuberculosis $\mathrm{H} 37 \mathrm{Ra}$, M. marinum ATCC BAA-535, and M. smegmatis str. MC2 155 were grown at $37^{\circ} \mathrm{C}$ (except for $M$. marinum; $30^{\circ} \mathrm{C}$ ) under rotating conditions, in Middlebrook 7H9 medium (Difco Laboratories, Detroit, MI, United States) containing $0.05 \%$ Tween 80 (Sigma-Aldrich, St. Louis, MO, United States) and 10\% Albumin-DextroseCatalase (Becton Dickinson and co., Sparks, MD, United States). Middlebrook 7H10 media was used as the solid medium supplemented with $10 \%$ Oleic acid-Albumin-Dextrose-Catalase (Becton Dickinson and co., Sparks, MD, United States). Yersinia 
enterocolitica DSMZ 23249 and E. coli were grown in LuriaBertani Medium (Difco) at $30^{\circ} \mathrm{C}$. As required, antibiotics were added as follows: $50 \mu \mathrm{g} \cdot \mathrm{ml}^{-1}$ kanamycin for E. coli and $Y$. enterocolitica and $25 \mu \mathrm{g} \cdot \mathrm{ml}^{-1}$ for Mycobacteria, $100 \mu \mathrm{g} \cdot \mathrm{ml}^{-1}$ hygromycin for E. coli and $50 \mu \mathrm{g} \cdot \mathrm{ml}^{-1}$ for Mycobacteria, $25 \mu \mathrm{g} \cdot \mathrm{ml}^{-1}$ chloramphenicol for E. coli and Y. enterocolitica, and $100 \mu \mathrm{g} . \mathrm{ml}^{-1}$ ampicillin for E. coli. All the strains used in this study are summarized in Table 1.

\section{Mycobacteria Plasmids Construction}

(1) Plasmid containing sigK-rskA full length: The construction of plasmids coding for sigK-rskA from M. tuberculosis (pMV306Hyg::dyad) and M. bovis (pMV::dyadbovis) have been already described (Said-Salim et al., 2006; Veyrier et al., 2008). To construct $\mathrm{pMV}::$ dyadoryx a part of the sigK-rskA region has been amplified, from genomic DNA of $M$. orygis, using Rv0444OF and Rv0444OR primers (Table 2). This amplicon was ligated to pMV306-Hyg::dyad digested with XhoI and HindIII (Figure 1A). Secondly, the reporter system was added to these plasmids: pLx-MPT70 (Veyrier et al., 2008) was digested using XbaI-SphI to extract $\operatorname{lux} A B$ fused to $m p t 70 p$. This fragment was treated with $\mathrm{T} 4$ polymerase to generate blunt ends and ligated with pMV306-Hyg::dyad, pMV::dyadbovis or pMV::dyadoryx digested by BsrDI. Doing that, pMV::dyadRvLx70, pMV::dyadbovis-Lx70 and pMV::dyadoryx-Lx70 have been respectively, generated.

(2) Plasmids coding for truncated versions of RskA: To construct all these plasmids a fragment of rskA from pMV::dyadbovis-Lx70 was removed after XhoI-HindIII digestion (Figure 1A), replaced by PCR products obtained using Pfu cloned polymerase (Stratagen Inc.) and digested with the same enzymes. These PCR products were obtained using Rv0444cOF and a specific reverse primer (Table 2). As an example, this was done using RskA1/121R which contained a HindIII site designed to introduce a stop codon in rskA after the 121 amino acids of RskA. In this case, the ligation of the PCR product obtained using $M$. tuberculosis gDNA template has generated pMV::dyad1/121Rv whereas the ligation of the PCR product obtained using the M. bovis gDNA template has generated pMV::dyad1/121bovis. Lastly, pMV::dyad $\Delta$ rskA was obtained by self ligation of pMV::dyadbovis-Lx70 digested with XhoI-HindIII and treated with $\mathrm{T} 4$ polymerase.

(3) pOE-Lx70 versions of the plasmids: To increase our signal we generated new version of luciferase expressing plasmids where the sigK-rskA are under the control of hsp60p. We digested pMV::dyadbovis-Lx70 with Pcil, treated with T4 DNA polymerase to generate blunt ends and further digested with HindIII. This product was ligated with $h s p 60 p$ from pMV261 digested with DraI and HindIII to generate pOE-Lx70 where hsp60p has replace the dyad. In order to re-incorporate the dyads, amplified the M. tuberculosis version with SigK3F and Rv0444HindIII primers (Table 2) and introduced it in pOE-Lx70 after a ligation of MunI-HindIII digestion products to obtain pOELx70DyadRv. All the other versions of RskA were reintroduced in this backbone plasmid as described above using XhoI-HindIII.

(4) Plasmids expressing SigK with serines instead of cysteines: To generate these constructs, site-directed mutagenesis on cysteine amino acids residues position 133 and 183 on SigK was performed, using three fragments PCR assembly method and specific primers SigK133F, SigK133R, and SigK183R (Table 2). PCR product was digested by MunI-HindIII and cloned into pOE-Lx plasmid digested by the same enzymes to generate pOELx SigKaloneSer. Then, rskA from M. tuberculosis, M. bovis and M. orygis, previously digested by XhoI-HindIII, were cloned into the generated pOE-Lx::DyadSerM.tb, pOE-Lx::DyadSerM.bo and pOE-Lx::DyadSerM.or.

(5) FLAG-tagged SigK: To generate the FLAG versions of SigK, we introduced a $1 \mathrm{X}$ FLAG-tag at the N-terminal part of sigK in both cysteine and serine version. Both versions of sigK were amplified from plasmids pOE-Lx::SigKalone and pOELx::SigKaloneSer by PCR using Phusion HF DNA polymerase (New England, BioLabs) and primers FlagSigKF and SigKR (Table 2). PCR products and pOE-Lx plasmids were digested by MunI-HindIII and ligated together. Then, $r s k A$ versions from M. tuberculosis, M. bovis and M. orygis were amplified from previous pOE-Lx plasmids, digested by XhoI-HindIII and ligated to pOE-Lx::SigKalone and pOE-Lx::SigKaloneSer to generate pOE-Lx::SigKaloneFlag with Dyad ${ }_{\text {tuberculosis }}$, Dyad $_{\text {bovis }}$ and Dyad $_{\text {orygis }}$. versus pOE-Lx::SigKaloneSerFlag with Dyad tuberculosis $_{\text {, }}$ Dyad $_{\text {bovis }}$ and Dyad orygis. $_{\text {. }}$

(6) Nocardia version of sigK-rskA: The locus from Nocardia asteroides 42007, N. nova 90960 or N. abscessus 91107 was amplified by the AccuPrime ${ }^{\mathrm{TM}}$ Taq DNA polymerase High Fidelity (Invitrogen), using NfRv3416F and NfRv3417R primers. These PCR products were cloned into TOPO using TOPO ${ }^{\circledR}$ TA Cloning ${ }^{\oplus}$ Kit (Invitrogen). The fragments were sequenced at the McGill University and Genome Quebec Innovation Center, and the sequence of the sigK locus from $N$. asteroides was deposited in GenBank (accession numbers: FJ935781). The TOPO plasmid comprising the N. asteroides locus was digested using BsaBI, ligated with pMV::dyadRv-Lx70 digested with HindIII-ApaLI (which removed the dyad from M. tuberculosis) and treated with $\mathrm{T} 4$ polymerase to obtain pMV::dyadN42Lx70. In a derivate plasmid called pMV::dyadN42stop-Lx70, a premature stop codon was inserted in $r s k A_{\text {nocardia }}$ by ligation of pre-annealed primers (PpuMIStopF and PpuMIStopR) with PpuMI digested pMV::dyadN42-Lx70.

All plasmids used in Mycobacteria (Table 1) were derived from pMV306 and were therefore integrated into the genomes of the specified mycobacteria. Plasmids were electroporated into M. smegmatis, M. tuberculosis $\mathrm{H} 37 \mathrm{Ra}$ and $\mathrm{H} 37 \mathrm{Rv}$, and M. marinum as previously described (Belley et al., 2004). Hygromycin resistant clones were verified by PCR and selected for further experiments.

\section{Luciferase Assays for Mycobacteria (LuXAB)}

As described before (Veyrier et al., 2008), bacteria were grown in $7 \mathrm{H} 9$ with hygromycin until the cultures reached an $\mathrm{OD}_{600}$ of 0.5 . After centrifugation, cultures were resuspended in PhosphateBuffered Saline (PBS) and $10 \mu \mathrm{l}$ of $1 \% \mathrm{n}$-decyl aldehyde (Sigma) in ethanol was added to $90 \mu \mathrm{l}\left(9 \times 10^{6}\right.$ bacteria $)$ in 96 well plates (Becton Dickinson labware). Light was immediately measured in 
TABLE 1 | Strains and plasmids used in this study.

\begin{tabular}{|c|c|c|}
\hline Strains or plasmids & Relevant genotype description & Reference \\
\hline \multicolumn{3}{|l|}{ Strains } \\
\hline E. coli DH5 $\alpha$ & Host for cloning & NEB \\
\hline E. coli TOP10 & Host for cloning & Invitrogen \\
\hline E. coli BL21 (DE3) pLysS & Host for expression vector & NEB \\
\hline M. smegmatis mc 155 & Lab strain & \\
\hline M. tuberculosis H37Ra & Lab strain & \\
\hline M. tuberculosis H37Rv & Human isolate & \\
\hline M. bovis 68799 & Bovine isolate & \\
\hline M. orygis 51145 & Antilope isolate & {$[44]$} \\
\hline Y. enterocolitica DSMZ 23249 & Lab strain & \\
\hline \multicolumn{3}{|l|}{ Plasmids } \\
\hline pMV306 & Integrase, $\mathrm{Hyg}^{r}$ & $\begin{array}{l}\text { Said-Salim } \\
\text { et al., } 2006\end{array}$ \\
\hline pMV306::DyadM.tb & Integrase, sigK and rskA of M. tuberculosis, $\mathrm{Hyg}^{\mathrm{r}}$ & $\begin{array}{l}\text { Said-Salim } \\
\text { et al., } 2006\end{array}$ \\
\hline pMV306::DyadM.bo & Integrase, $\mathrm{Hyg}^{r}$, sigK of $M$. tuberculosis and rskA of $M$. bovis & This study \\
\hline pMV306::DyadM.or & Integrase, $\mathrm{Hyg}^{\mathrm{r}}$, sigK of $\mathrm{M}$. tuberculosis and rskA of $M$. orygis & This study \\
\hline pLx-MPT70 & I $u x A B$ genes under mpt70 promoter, replication origin E.coli, $\mathrm{Hyg}^{\mathrm{r}}$ & $\begin{array}{l}\text { Veyrier et al., } \\
2008\end{array}$ \\
\hline pMV306-Lx & Integrase, luxAB genes under mpt70 promoter, $\mathrm{Hyg}^{\mathrm{r}}$ & This study \\
\hline pOE-Lx & Integrase, luxAB under mpt70 promoter, hsp60 promoter, $\mathrm{Hyg}^{\mathrm{r}}$ & This study \\
\hline pET28b+ & Replication origin E.coli, T7 promoter, $\mathrm{Km}^{r}$ & Novagen \\
\hline pMV306-Lx::SigKalone & sigk of M. tuberculosis & This study \\
\hline pMV306-Lx::DyadM.tb & sigk and rskA of M. tuberculosis & This study \\
\hline pMV306-Lx::DyadM.bo & sigk of $M$. tuberculosis and rskA of $M$. bovis & This study \\
\hline pMV306-Lx::DyadM.or & sigk of $M$. tuberculosis and rskA of $M$. orygis & This study \\
\hline pMV306-Lx::Dyad1/193M.tb & sigK and rskA 1/193 of M. tuberculosis & This study \\
\hline pMV306-Lx::Dyad1/193M.bo & sigk of $M$. tuberculosis and rskA 1/193 of M. bovis & This study \\
\hline pMV306-Lx::Dyad1/86M.tb & sigk and rskA 1/86 of M. tuberculosis & This study \\
\hline pOE-Lx::SigKalone & sigk of $M$. tuberculosis & This study \\
\hline pOE-Lx::DyadM.tb & sigk and rskA of $M$. tuberculosis & This study \\
\hline pOE-Lx::DyadM.bo & sigk of $M$. tuberculosis and rskA of $M$. bovis & This study \\
\hline pOE-Lx::DyadM.or & sigk of $M$. tuberculosis and rskA of $M$. orygis & This study \\
\hline pOE-Lx::SigKaloneSer & sigK C133S - C183S of M. tuberculosis & This study \\
\hline pOE-Lx::DyadSerM. tb & sigK C133S - C183S and rskA of M. tuberculosis & This study \\
\hline pOE-Lx::DyadSerM.bo & sigk C133S - C183S of M. tuberculosis and rskA of M. bovis & This study \\
\hline pOE-Lx::DyadSerM.or & sigK C133S - C183S of $M$. tuberculosis and rskA of $M$. orygis & This study \\
\hline pOE-Lx::SigKaloneFlag & sigK FLAG-tag in N-terminal of $M$. tuberculosis & This study \\
\hline pOE-Lx::DyadM.tbFlag & sigK FLAG-tag in N-terminal and rskA of $M$. tuberculosis & This study \\
\hline pOE-Lx::DyadM.boFlag & sigK FLAG-tag in N-terminal of $M$. tuberculosis and rskA of $M$. bovis & This study \\
\hline pOE-Lx::DyadM.orFlag & sigK FLAG-tag in N-terminal of $M$. tuberculosis and rskA of $M$. orygis & This study \\
\hline pOE-Lx::SigKaloneSerFlag & sigK C133S - C183S FLAG-tag in N-terminal of M. tuberculosis & This study \\
\hline pOE-Lx::DyadSerM.tbFlag & sigK C133S - C183S FLAG-tag in N-terminal and rskA of $M$. tuberculosis & This study \\
\hline pOE-Lx::DyadSerM.boFlag & sigK C133S - C183S FLAG-tag in N-terminal of $M$. tuberculosis and rskA of $M$. bovis & This study \\
\hline pOE-Lx::DyadSerM.orFlag & sigK C133S - C183S FLAG-tag in N-terminal of $M$. tuberculosis and rskA of $M$. orygis rskA of $M$. tuberculosi & This study \\
\hline pKO5luc & luc gene under mpt83 promoter, $\mathrm{Cm}^{r}$ & This study \\
\hline pBAD-Km::SigK & sigK of Y. enterocolitica, $\mathrm{Km}^{\mathrm{r}}$ & This study \\
\hline pBAD-Km::SigK-RskA & sigK and $r$ skA of $Y$. enterocolitica, $\mathrm{Km}^{\mathrm{r}}$ & This study \\
\hline pBAD-Km::SigK-RskA179 & sigK and rskA 1/179 of Y. enterocolitica, $\mathrm{Km}^{\mathrm{r}}$ & This study \\
\hline pBAD-Km::SigK-RskA85 & sigK and rskA 1/85 of $Y$. enterocolitica, $\mathrm{Km}^{r}$ & This study \\
\hline pBAD-Km::SigKC181S & sigK C181S of $Y$. enterocolitica, $\mathrm{Km}^{\mathrm{r}}$ & This study \\
\hline pBAD-Km::SigKC181S-RskA & sigK C181S and rskA of Y. enterocolitica, $\mathrm{Km}^{\mathrm{r}}$ & This study \\
\hline pBAD-Km::SigKC181S-RskA179 & sigK C181S and rskA 1/179 of Y. enterocolitica, $\mathrm{Km}^{r}$ & This study \\
\hline pBAD-Km::SigKC181S-RskA85 & sigK C181S and rskA 1/85 of Y. enterocolitica, $\mathrm{Km}^{\mathrm{r}}$ & This study \\
\hline pET28a::SigK & sigK of $Y$. enterocolitica, $\mathrm{Km}^{\mathrm{r}}$, His-tag in sigK N-terminal & This study \\
\hline pET28a::SigK-RskA & sigK and rskA of $Y$. enterocolitica, $\mathrm{Km}^{r}$, His-tag in sigK $\mathrm{N}$-terminal & This study \\
\hline pET28a::SigK-RskA179 & sigK and rskA 1/179 of $Y$. enterocolitica, $\mathrm{Km}^{r}$, His-tag in sigK $\mathrm{N}$-terminal & This study \\
\hline pET28a::SigK-RskA85 & sigK and rskA 1/85 of $Y$. enterocolitica, $\mathrm{Km}^{r}$, His-tag in sigK $\mathrm{N}$-terminal & This study \\
\hline
\end{tabular}


TABLE 2 | Oligonucleotide primers used in this study.

\begin{tabular}{|c|c|}
\hline Primers name & Sequence $\left(5^{\prime} \rightarrow 3^{\prime}\right)$ \\
\hline Rv0444OF & GTGCTGCAGGGTGCGGCCAACG \\
\hline Rv0444OR & GGAAAGCTITGATAACGGCGACATC \\
\hline RskA1/46R & CTCA_AGCTTAGTCGTTGAAAGCCGC \\
\hline RskA1/86R & GCCAAGCTTCAGCGTGATTGGCGC \\
\hline RskA1/121R & GCT $\underline{A A G C T T C G G T G G G G G G T G G ~}$ \\
\hline RskA1/193R & CCGAAGCTTAGGGCGTCACCGCC \\
\hline RskA71/232F & CATCTCGAGACGGCCATCCTGGAT \\
\hline NfRv3416F & GACGTCTGGGACTGGCAGATGC \\
\hline NfRv3417R & CGCATGGTGCTCACCACCGAG \\
\hline PpuMIStopF & GACTAGTAAGTGAGTAGGGC \\
\hline PpuMIStopR & GTCGCCCTACTCACTTACTA \\
\hline SigAMsF & CCAAGGGCTACAAGTTCTCG \\
\hline SigAMsR & TGGATCTCCAGCACCTTCTC \\
\hline SigKMunIF & GACCCAATTGACCATCACGGCGC \\
\hline Rv0444RHindIII & ATAAAAGCTTCCGGCGTGTTCGTCGCGATGC \\
\hline SigK133F & CGCCGGGTGACCGAGTCCCTCAAGGCGTTGACCGAC \\
\hline SigK133R & GTCGGTCAACGCCTTGAGGGACTCGGTCACCCGGCG \\
\hline SigK183R & $\begin{array}{l}\text { GGGAAGCTTACTCGAGCAGCTCAAAATCG } \\
\text { GTATGTTCAGTCATGAGCGCCGCTCTCCCAAC } \\
\text { GCATCGCTTCGCTCGGCCGGCGCAGTCATGACAC } \\
\text { GTCCAGGGAGTTGCGCAGGCTGCGC }\end{array}$ \\
\hline FlagSigKF & $\begin{array}{l}\text { AAGACAATTGAATGGACTACAA } \\
\text { GGACGACGATGACAAGACCGGACCGCCACGGCTGAGC }\end{array}$ \\
\hline SigKR & GGGAAGCTTACTCGAGCAGCTCAAAATCGG \\
\hline RskAMtb_F & ATATATAAAGCTTATGACTGAACATACCGATITGAGC \\
\hline RskAMtb_R & ATATGCGGCCGCTCACCCGAGCGGCAGCT \\
\hline 5'KOtriadYEF & GCAGCGGCCGCCGATाTGCTGAAATTG \\
\hline P83YER & CATAATGTATTCCTGTAGGTTGAGGTG \\
\hline P83LucF & $\begin{array}{l}\text { CCTCAACCTACAGGAATACATTATGGAAGACGCCAA } \\
\text { AAACATAAAG }\end{array}$ \\
\hline LucXholR & GCACTCGAGCCTACAATTTGGACПTCC \\
\hline SigKYeBspHIF & GGCTCATGAATGAATGTTCTGTGGAAC \\
\hline SigKYeXbalR & GAGTCTAGAGTCATATTCGCATCTGTITTC \\
\hline RskAYeXbalR & ATGTCTAGACTCCGGTGGAAAAAGTCTTAC \\
\hline RskAL180*WAR & GACTCTAGACTTATTGTGTCGGCCCTTGAGTATTC \\
\hline RskAW86*R & $\begin{array}{l}\text { GGCTCTAGATTAAGGGTICGCTTCA } \\
\text { TGTGGCGGATATTGATCGGTGGTAACTGCAACTCCAG } \\
\text { CCGTICCAAACGCGCTCAGGTGG }\end{array}$ \\
\hline SigKC181SR & $\begin{array}{l}\text { CATATTCGCATCTGTIITCATAAGCC } \\
\text { TACAGAGTCCTITAGGTGGTCC }\end{array}$ \\
\hline SigKC181SF & $\begin{array}{l}\text { GGACCACCTAAAGGACTCTGTAGGCTT } \\
\text { ATGAAAAACAGATGCGAATATG }\end{array}$ \\
\hline SigKYeNdelF & GGCATATGGATGAATGTTCTGTGGAAC \\
\hline SigKYeBamHIR & GGATCCTAGAGTCATATTCGCATCTGTIITC \\
\hline
\end{tabular}

the Victor $3^{\mathrm{TM}}$ Wallac 1420 Multilabel Counter (PerkinElmer) over a $10 \mathrm{~s}$ period. For each experiment, 2 transformants have been measured two times and luminescence output was expressed in RLU (Relative Light Unit).

To test expression of luciferase in bacteria harboring the transposon, bacteria were grown overnight in $1 \mathrm{ml}$ of $7 \mathrm{H} 9$ with hygromycin and kanamycin. After centrifugation, cultures were resuspended in Phosphate-Buffered Saline (PBS) and tested as described above. In this case, we normalized the amount of light with the $\mathrm{OD}_{600}$ measured in the 96 well plates with the Victor $3^{\mathrm{TM}}$ Wallac 1420 Multilabel Counter (PerkinElmer).

\section{RNA Extraction and Quantitative RT-qPCR}

The aim was to determine the expression of sigK $K_{\text {tuberculosis }}$ in $M$. smegmatis harboring the different plasmids. To do that we performed RT-qPCR and normalized against the RNA amount of sigA $A_{\text {smegmatis }}$ (Manganelli et al., 1999). RNA was extracted from cultures with an $\mathrm{OD}_{600}$ of 0.4 to 0.6 by a modified phenolchloroform extraction method (Charlet et al., 2005). The RTqPCR methods as well as the primers used for sigK have already been described (Charlet et al., 2005) except for the primers (SigAMsF and SigAMsR) used for sig $A_{\text {smegmatis }}$ which are listed in Table 2.

\section{Protein Preparation and Immunoblot}

For secreted protein (MPT70), $10 \mathrm{ml}$ of culture supernatants of indicated $M$. tuberculosis strains were concentrated using a Millipore Ultra-10 Centrifugal Filter Unit (10 000 MW cutoff) and filtered through a $0.22 \mu \mathrm{m}$ membrane. For membrane associated proteins (MPT70 and MPT83), culture pellet of a $10 \mathrm{ml}$ culture was resuspended in $1 \mathrm{ml}$ of water and samples were subjected to FastPrep (BIO 101 Savant) for $15 \mathrm{~s}$ at $6.0 \mathrm{rpm} 3$ times. To verify the expression of SigK, single colonies from M. smegmatis::sigK, M. smegmatis::dyadtub, $M$. smegmatis::dyadbov, and M. smegmatis::dyadoryx, as well as its mutated versions (C133S and C183S) were picked, inoculated into $2 \mathrm{~mL}$ of $7 \mathrm{H} 9$ medium supplemented with $50 \mu \mathrm{g} \mathrm{mL}-1$ hygromycin and incubated $48 \mathrm{~h}$ at $37^{\circ} \mathrm{C}, 250 \mathrm{rpm}$. Bacteria harvesting was done by centrifugation of $1 \mathrm{~mL}$ of culture at $8,000 \times g$, RT, $10 \mathrm{~min}$. Cell pellets were resuspended in $200 \mu \mathrm{L}$ $1 \mathrm{X}$ PBS. Lysis was performed by addition of $65 \mu \mathrm{L}$ of $4 \mathrm{X}$ Laemmli sample buffer, followed by boiling at $100^{\circ} \mathrm{C}$ for $10 \mathrm{~min}$. Samples were run on a $12 \%$ SDS-PAGE gel for $1 \mathrm{~h}$ at $200 \mathrm{~V}$.

For blotting assay, protein transfer was performed on PVDF membranes (BioRad) at $80 \mathrm{~V}$ for $2 \mathrm{~h}$. Membrane blocking was carried out for $1 \mathrm{~h}$ at room temperature, using TBSTween $0.1 \%$ containing 5\% BSA (TBS-TB). Incubation with rabbit polyclonal antibodies against MPT70 and MPT83 (gifts of Harald Wiker) (1:500 dilution), mouse monoclonal antibodies against HSP65 (Abcam), Mouse monoclonal DYKDDDDK Tag Antibody (FG4R) and Mouse RpoB Monoclonal Antibody (8RB13) were blotted onto the PVDF membrane overnight at $4^{\circ} \mathrm{C}$ followed by $1 \mathrm{~h}$ incubation with anti-rabbit $\operatorname{IgG}-$ horseradish peroxidase conjugate (1:15 000 dilution) or anti-mouse IgGhorseradish peroxidase conjugate (1:15 000 dilution). Three washing steps with TBS-Tween $0.1 \%$ of 10 min each were added after incubation with both antibodies. Proteins were visualized by ECL, using the substrate SuperSignal West Pico (Thermo Fisher Scientific).

\section{Enterobacterales Plasmids Construction}

(1) pKO5'Luc: This plasmid, with a pHS1 (a temperaturesensitive derivative of pSC101) origin of replication, was used to express the firefly luciferase under the control of 
mpt83p from $Y$. enterocolitica. To construct this plasmid the luciferase was fused to mpt83p by a 3 step PCR using the primers P83LucF/LucXhoIR to amplify the luciferase and 5 'KOtriadYEF/P83YER (Table 2) to amplify the promoter. The fused PCR product were digested with NotI-XhoI and cloned in pKO3 (Link et al., 1997) digested with NotI-SalI to give pKO5Luc.

(2) pBAD::Km with sigK and rskA from Y. enterocolitica: Coding sequences for SigK, SigK-RskA, SigK-RskA179 and SigKRskA85 were amplified using specific primers. In all cases, the forward primer was SigKYeBspHIF, and the reverse primers were SigKYeXbaIR, RskAYeXbaIR, RskAWA179*R, and RskAW85*R, respectively. Restriction sites for $\mathrm{BspHI}$ (forward primer) and $\mathrm{XbaI}$ (reverse primer) were used to clone into pBAD digested with NcoI and XbaI.

(3) pBADSigKmut: For SigKC181S mutant, sigK was amplified using the forward primer mentioned above, and SigKC181SR. Different variants of $r s k A$ were amplified using the reverse primers already described, and the forward primer SigKC181SF, which overlaps with SigKC181SR, allowing the fusion with sigKC181S and generating the variants sigKC181SrskA, sigKC181S-rskA179 and sigKC181S-rskA85. The PCR products were digested with $\mathrm{BspHI}$ and $\mathrm{XbaI}$ and cloned into $\mathrm{pBAD}-\mathrm{Km}$ vector digested with $\mathrm{NcoI}$ and $\mathrm{XbaI}$.

(4) pET28a::SigK: SigK was cloned into pET28a(+) vector using primers SigKYeNdeIF and SigKYeBamHIR. Both PCR product and vector were digested by NdeI and BamHI for introduction of a $\mathrm{N}$-terminal $6 \mathrm{xHis}$. After ligation, constructs were cloned in E. coli $\mathrm{DH} 5 \alpha$ and then transformed in E. coli BL21(DE3) for protein expression.

\section{Luciferase Assays for Enterobacterales (Firefly Luciferase)}

For the in vitro luciferase assay, bacteria co-transformed with $\mathrm{pBAD}-\mathrm{Km}$ vector containing the different SigK-RskA constructions and $\mathrm{pKO} 5^{\prime} \mathrm{luc}$, were grown overnight at $30^{\circ} \mathrm{C}$, $200 \mathrm{rpm}$, in $\mathrm{LB}$ medium containing $50 \mu \mathrm{g} \mathrm{\textrm {mL } ^ { - 1 }}$ kanamycin and $25 \mu \mathrm{g} \mathrm{mL}^{-1}$ chloramphenicol. From the overnight cultures, $100 \mu \mathrm{L}$ were inoculated into $3 \mathrm{~mL} \mathrm{LB}$ with the antibiotics already mentioned. In a 96 well microplate, $67.5 \mu \mathrm{L}$ of each fresh culture were dispensed in triplicate considering two conditions: induction and non-induction. For induction condition, 0.06\% $\mathrm{L}$-arabinose was added. Therefore, the plate included bacteria expressing the different versions of SigK-RskA (wild-type and mutant), as well as bacteria expressing the empty vector (pBAD$\mathrm{Km}$, as a negative control), both in absence and presence of $\mathrm{L}$-arabinose. The induction was carried out for the indicated time at $30^{\circ} \mathrm{C}$. Then, $75 \mu \mathrm{L}$ of the Luciferase Assay Reagent (Promega) were added in each well, and the luminescence was measured using a Wallac Victor 3 (Perkin Elmer) luminometer. Luminescence was normalized by the bacterial OD determined by absorbance at $600 \mathrm{~nm}$.

\section{SigK Overexpression and Purification From E. coli}

From an overnight culture, $10 \mathrm{~mL}$ were inoculated into $1 \mathrm{~L}$ of LB medium supplemented with $50 \mu \mathrm{g} \mathrm{mL}-1$ kanamycin and grown at $37^{\circ} \mathrm{C}, 250 \mathrm{rpm}$ until reaching an $\mathrm{OD}_{600}$ of 0.6 . Induction was performed with $1 \mathrm{mM}$ isopropyl $\beta$-D-1thiogalactopyranoside (IPTG) at $37^{\circ} \mathrm{C}$ for $4 \mathrm{~h}$. Bacteria harvesting was carried out by centrifugation at $10,000 \mathrm{rpm}, 4^{\circ} \mathrm{C}, 15 \mathrm{~min}$. Cell pellets were resuspended in $20 \mathrm{~mL}$ native binding buffer $\left(50 \mathrm{mM} \mathrm{NaH} \mathrm{PO}_{4}, 300 \mathrm{mM} \mathrm{NaCl}\right.$, and $10 \mathrm{mM}$ imidazole, $\mathrm{pH}$ 8). Pellets were kept on ice, treated with $1 \mathrm{mg} \mathrm{mL}^{-1}$ lysozyme and $1 \mathrm{mM}$ PMSF for $30 \mathrm{~min}$, and lysed by sonication. Lysates were clarified by centrifugation at $15,000 \mathrm{rpm}, 4^{\circ} \mathrm{C}, 30 \mathrm{~min}$, and purified using HisPur ${ }^{\mathrm{TM}}$ Ni-NTA Resin (Thermo Fisher Scientific). Lysates were loaded onto the purification column (previously equilibrated with native binding buffer), unspecific binding was removed by washing ( $50 \mathrm{mM} \mathrm{NaH} 2 \mathrm{PO} 4,300 \mathrm{mM}$ $\mathrm{NaCl}$, and $20 \mathrm{mM}$ imidazole), and proteins were eluted (50 $\mathrm{mM}$ $\mathrm{NaH} 2 \mathrm{PO} 4,300 \mathrm{mM} \mathrm{NaCl}$, and $150 \mathrm{mM}$ imidazole). Protein identity was confirmed by western blotting using a monoclonal Anti-His IgG1 - from mouse (Sigma).

\section{SigK Western Blotting}

To verify the expression of SigK, single colonies from E. coli BL21(DE3)::sigK, E. coli BL21(DE3)::sigK-rskA, E. coli BL21(DE3)::sigK-rskA182 and E. coli BL21(DE3)::sigK-rskA81 were picked, inoculated into $5 \mathrm{~mL}$ of $\mathrm{LB}$ supplemented with $50 \mu \mathrm{g} \mathrm{mL}^{-1}$ kanamycin and incubated overnight at $37^{\circ} \mathrm{C}$, $250 \mathrm{rpm}$. The overnight cultures were diluted into $500 \mathrm{~mL}$ LB supplemented with $50 \mu \mathrm{g} \mathrm{mL} \mathrm{m}^{-1}$ kanamycin, and incubated at $37^{\circ} \mathrm{C}, 250 \mathrm{rpm}$, until $\mathrm{OD}_{600}$ reached 0.6. Induction was performed as described before. Bacteria harvesting was done by centrifugation at $10,000 \mathrm{rpm}, 4^{\circ} \mathrm{C}, 15 \mathrm{~min}$. Cell pellets were resuspended in $10 \mathrm{~mL} 1 \mathrm{X}$ PBS containing $0.5 \%$ SDS, kept on ice and lysed by sonication. Lysates were clarified as previously indicated. Then, $5 \mu \mathrm{L}$ of each sample were diluted into $7 \mu \mathrm{L} \mathrm{H}_{2} \mathrm{O}$ and $4 \mu \mathrm{L}$ of $4 \mathrm{X}$ Laemmli sample buffer (0.2 M Tris pH 6.8, 0.08\% w/v SDS, $0.2 \%$ v/v $\beta$-mercaptoethanol, $0.4 \%$ v/v Glycerol, $0.002 \%$ $\mathrm{w} / \mathrm{v}$ Bromophenol blue), and boiled at $100^{\circ} \mathrm{C}$ for $10 \mathrm{~min}$. Samples were run on a $12 \%$ SDS-PAGE gel for $1 \mathrm{~h}$ at $200 \mathrm{~V}$.

For blotting assay, protein transfer was performed on PVDF membranes (BioRad) at $90 \mathrm{~V}$ for $1 \mathrm{~h} 30 \mathrm{~min}$. Membrane blocking was carried out for $1 \mathrm{~h}$ at room temperature, using TBS-Tween $0.1 \%$ containing 5\% BSA (TBS-TB). Incubation with 1:500 in TBS-TB of primary antibody (monoclonal Anti-His IgG1 from mouse) was performed overnight at $4^{\circ} \mathrm{C}$. An anti-mouse IgG - HRP conjugate - from rabbit was used as a secondary antibody (1:10000 in TBS-TB), incubating for $1 \mathrm{~h}$ at RT. Three washing steps with TBS-Tween $0.1 \%$ of 10 min each were added after incubation with both antibodies. Proteins were visualized by ECL, using the substrate SuperSignal West Pico (Thermo Fisher Scientific).

RpoB detection [by incubation with an RNA polymerase beta Monoclonal Antibody (8RB13) - from mouse], was used as loading control.

\section{SigK and RskA in silico Study}

The sequences of SigK from $M$. tuberculosis and Yersinia enterocolitica were added to a database constituted by another study and containing 4352 sequences of ECF Sigma factors (Pinto and da Fonseca, 2020). CD-HIT version 4.8.1 (Fu et al., 2012) 
was used to keep only one representative sequence for those sharing $95 \%$ or more of identity. The resulting 3717 sequences were aligned using MAFFT version 7.453 using 25 refinement iterations (Katoh and Standley, 2013). A phylogenetic reconstruction was then performed using FastTree version 2.1.11 (Price et al., 2010) with the LG+CAT model. Finally, the tree was visualized using FigTree version 1.4.3 ${ }^{2}$.

The sequences of representative genomes of the RefSeq database have been downloaded (Supplementary Table S1). Subsequently, sequences homologous to SigK were searched in the set of coding sequences using BLASTP version 2.10.0. The sequences having an e-value and a percentage of similarity greater than or equal to $1 \mathrm{e}-10$ and $50 \%$, respectively, were retained. The RskA homologous sequences were also searched by BLASTP, with the default parameters, but with the obligation that the RskA be adjacent to the identified SigK, considering the polarity of the genes. In the event that no RskA was found, the adjacent gene was still extracted and investigated manually by annotation and COG categorization using EggNOG mapper (Huerta-Cepas et al., 2017).

The 4157 SigK sequences were then aligned by MAFFT with 25 iterations. The amino acids at positions 133 and 183 according to the sequence of $M$. tuberculosis were extracted in order to verify the conservation of the cysteines. Different sequence characteristics, such as the number of total cysteines, CXXC (zinc finger) and length were determined using a custom python script. Finally, the nucleotide sequences of the RskAs encoded as pseudogenes in RefSeq for the genus Nocardia have been downloaded, translated into amino acids and their length calculated with an in-house script.

\section{DATA AVAILABILITY STATEMENT}

The datasets presented in this study can be found in online repositories. The names of the repository/repositories and accession number(s) can be found in the article/ Supplementary Material.

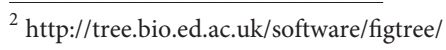

\section{REFERENCES}

Bastiaansen, K. C., van Ulsen, P., Wijtmans, M., Bitter, W., and Llamas, M. A. (2015). Self-cleavage of the Pseudomonas aeruginosa cell-surface signaling antisigma factor FoxR occurs through an N-O Acyl rearrangement. J. Biol. Chem. 290, 12237-12246. doi: 10.1074/jbc.m115.643098

Belley, A., Alexander, D., Di Pietrantonio, T., Girard, M., Jones, J., Schurr, E., et al. (2004). Impact of methoxymycolic acid production by Mycobacterium bovis BCG vaccines. Infect. Immun. 72, 2803-2809. doi: 10.1128/iai.72.5.2803-2809. 2004

Brites, D., Loiseau, C., Menardo, F., Borrell, S., Boniotti, M. B., Warren, R., et al. (2018). A new phylogenetic framework for the animal-adapted Mycobacterium tuberculosis complex. Front. Microbiol. 9:2820. doi: 10.3389/fmicb.2018. 02820

Brooks, B. E., and Buchanan, S. K. (2007). Signaling mechanisms for activation of extracytoplasmic function (ECF) sigma factors. Biochim. Biophys. Acta 1778, 1930-1945. doi: 10.1016/j.bbamem.2007.06.005

\section{AUTHOR CONTRIBUTIONS}

FV and MB designed the study. FV, CN, LL, HT, and AV performed and analyzed the experiments. FV, CN, AV, and $\mathrm{MB}$ wrote and edited the manuscript. All authors have read and approved the manuscript.

\section{FUNDING}

This work was supported by the FV Natural Sciences and Engineering Research Council of Canada (NSERC) under Grant RGPIN-2016-04940 and a CIHR Foundation Grant FDN148362. CN received Ph.D. studentship from the Institut Pasteur. AV received a Postdoctoral Fellowship from the NSERC. FV was a research scholar of the Fonds de Recherche du Québec-Santé. $\mathrm{MB}$ was supported by a Tier 1 Canada Research Chair.

\section{SUPPLEMENTARY MATERIAL}

The Supplementary Material for this article can be found online at: https://www.frontiersin.org/articles/10.3389/fmicb. 2020.558166/full\#supplementary-material

FIGURE S1 | Alignment (A) and schematic representations (B) of RskA versions used in this study (natural or engineered variants).

FIGURE S2 | (A) Effect of FLAG-tag linked to the N-terminus part of SigK was measured by luciferase under the control of mpt70p in $M$. smegmatis (B) Effect of His-tag linked to the $\mathrm{N}$-terminus part of SigK was measured by luciferase under the control of mpt83p in E. coli. Error bars represent standard deviations of 3 independent experiments with 2 different clones each time.

FIGURE S3 | Expression of SigK regulon through MPT70 antigen production was detected by immunoblot of $M$. tuberculosis $\Delta$ sigK cell extract. Dyad M.tb cysteines vs. serines version; Dyad M.bo cysteines vs. serines version; Dyad M.or cysteines vs. serines version.

TABLE S1 | Table presenting the in-silico analyses of SigK homologues using RefSeq database.

Charlet, D., Mostowy, S., Alexander, D., Sit, L., Wiker, H. G., and Behr, M. A. (2005). Reduced expression of antigenic proteins MPB70 and MPB83 in Mycobacterium bovis BCG strains due to a start codon mutation in sigK. Mol. Microbiol. 56, 1302-1313. doi: 10.1111/j.1365-2958.2005.04618.x

Fu, L., Niu, B., Zhu, Z., Wu, S., and Li, W. (2012). CD-HIT: accelerated for clustering the next-generation sequencing data. Bioinformatics 28, 3150-3152. doi: 10.1093/bioinformatics/bts565

Garnier, T., Eiglmeier, K., Camus, J. C., Medina, N., Mansoor, H., Pryor, M., et al. (2003). The complete genome sequence of Mycobacterium bovis. Proc. Natl. Acad. Sci. U.S.A. 100, 7877-7882. doi: 10.1073/pnas.1130426100

Gruber, T. M., and Gross, C. A. (2003). Multiple sigma subunits and the partitioning of bacterial transcription space. Annu. Rev. Microbiol. 57, 441-466. doi: 10.1146/annurev.micro.57.030502.090913

Helmann, J. D. (2002). The extracytoplasmic function (ECF) sigma factors. Adv. Microb. Physiol. 46, 47-110. doi: 10.1016/s0065-2911(02)46002-x

Huerta-Cepas, J., Forslund, K., Coelho, L. P., Szklarczyk, D., Jensen, L. J., von Mering, C., et al. (2017). Fast genome-wide functional annotation through 
orthology assignment by eggNOG-Mapper. Mol. Biol. Evol. 34, 2115-2122. doi: 10.1093/molbev/msx148

Igoshin, O. A., Brody, M. S., Price, C. W., and Savageau, M. A. (2007). Distinctive topologies of partner-switching signaling networks correlate with their physiological roles. J. Mol. Biol. 369, 1333-1352. doi: 10.1016/j.jmb.2007. 04.021

Katoh, K., and Standley, D. M. (2013). MAFFT multiple sequence alignment software version 7: improvements in performance and usability. Mol. Biol. Evol. 30, 772-780. doi: 10.1093/molbev/mst010

Link, A. J., Phillips, D., and Church, G. M. (1997). Methods for generating precise deletions and insertions in the genome of wild-type Escherichia coli: application to open reading frame characterization. J. Bacteriol. 179, 6228-6237. doi: 10. 1128/jb.179.20.6228-6237.1997

Lipworth, S., Jajou, R., de Neeling, A., Bradley, P., van der Hoek, W., Maphalala, G., et al. (2019). SNP-IT tool for identifying subspecies and associated lineages of Mycobacterium tuberculosis complex. Emerg. Infect. Dis. 25, 482-488. doi: 10.3201/eid2503.180894

Llamas, M. A., van der Sar, A., Chu, B. C., Sparrius, M., Vogel, H. J., and Bitter, W. (2009). A Novel extracytoplasmic function (ECF) sigma factor regulates virulence in Pseudomonas aeruginosa. PLoS Pathog. 5:e1000572. doi: 10.1371/ journal.ppat.1000572

Lonetto, M. A., Brown, K. L., Rudd, K. E., and Buttner, M. J. (1994). Analysis of the Streptomyces coelicolor sigE gene reveals the existence of a subfamily of eubacterial RNA polymerase sigma factors involved in the regulation of extracytoplasmic functions. Proc. Natl. Acad. Sci. U.S.A. 91, 7573-7577. doi: 10.1073/pnas.91.16.7573

Makinoshima, H., and Glickman, M. S. (2005). Regulation of Mycobacterium tuberculosis cell envelope composition and virulence by intramembrane proteolysis. Nature 436, 406-409. doi: 10.1038/nature03713

Malkhed, V., Gudlur, B., Kondagari, B., Dulapalli, R., and Vuruputuri, U. (2011). Study of interactions between Mycobacterium tuberculosis proteins: SigK and anti-SigK. J. Mol. Model. 17, 1109-1119. doi: 10.1007/s00894-0100792-7

Manganelli, R. (2014). Sigma factors: key molecules in Mycobacterium tuberculosis physiology and virulence. Microbiol. Spectr. 2:MGM2-0007-2013. doi: 10.1128/ microbiolspec.MGM2-0007-2013

Manganelli, R., Dubnau, E., Tyagi, S., Kramer, F. R., and Smith, I. (1999). Differential expression of 10 sigma factor genes in Mycobacterium tuberculosis. Mol. Microbiol. 31, 715-724. doi: 10.1046/j.1365-2958.1999. 01212.x

Mao, X. M., Sun, N., Wang, F., Luo, S., Zhou, Z., Feng, W. H., et al. (2013). Dual positive feedback regulation of protein degradation of an extra-cytoplasmic function sigma factor for cell differentiation in Streptomyces coelicolor. J. Biol. Chem. 288, 31217-31228. doi: 10.1074/jbc.M113.491498

Morris, R. S., Pfeiffer, D. U., and Jackson, R. (1994). The epidemiology of Mycobacterium bovis infections. Vet. Microbiol. 40, 153-177. doi: 10.1016/ 0378-1135(94)90053-1

Mostowy, S., and Behr, M. A. (2005). The origin and evolution of Mycobacterium tuberculosis. Clin. Chest. Med. 26, 207-216. doi: 10.1016/j.ccm.2005.02.004

Mostowy, S., Inwald, J., Gordon, S., Martin, C., Warren, R., Kremer, K., et al. (2005). Revisiting the evolution of Mycobacterium bovis. J. Bacteriol. 187, 6386-6395. doi: $10.1128 /$ jb.187.18.6386-6395.2005

Ochs, M., Angerer, A., Enz, S., and Braun, V. (1996). Surface signaling in transcriptional regulation of the ferric citrate transport system of Escherichia coli: mutational analysis of the alternative sigma factor FecI supports its essential role in fec transport gene transcription. Mol. Gen. Genet. 250, 455-465. doi: $10.1007 /$ bf02174034

Ochs, M., Veitinger, S., Kim, I., Welz, D., Angerer, A., and Braun, V. (1995). Regulation of citrate-dependent iron transport of Escherichia coli: fecR is required for transcription activation by FecI. Mol. Microbiol. 15, 119-132. doi: 10.1111/j.1365-2958.1995.tb02226.x

O’Reilly, L. M., and Daborn, C. J. (1995). The epidemiology of Mycobacterium bovis infections in animals and man: a review. Tubercle Lung Dis. 76(Suppl. 1), 1-46. doi: 10.1016/0962-8479(95)90591-x

Otero-Asman, J. R., Wettstadt, S., Bernal, P., and Llamas, M. A. (2019). Diversity of extracytoplasmic function sigma ( $\sigma$ ECF) factor-dependent signaling in Pseudomonas. Mol. Microbiol. 112, 356-373. doi: 10.1111/mmi.14331
Pinto, D., and da Fonseca, R. R. (2020). Evolution of the extracytoplasmic function $\sigma$ factor protein family. NAR Genom. Bioinform. 2:lqz026. doi: 10.1093/nargab/ lqz026

Pinto, D., and Mascher, T. (2016). "The ECF classification: a phylogenetic reflection of the regulatory diversity in the extracytoplasmic function $\sigma$ factor protein family," in Stress and Environmental Regulation of Gene Expression and Adaptation in Bacteria, ed. F. J. de Bruijn (Hoboken, NJ: John Wiley \& Sons, Inc), 64-96. doi: 10.1002/9781119004813.ch7

Price, M. N., Dehal, P. S., and Arkin, A. P. (2010). FastTree 2-approximately maximum-likelihood trees for large alignments. PLoS One 5:e9490. doi: 10. 1371/journal.pone.0009490

Riojas, M. A., McGough, K. J., Rider-Riojas, C. J., Rastogi, N., and Hazbon, M. H. (2018). Phylogenomic analysis of the species of the Mycobacterium tuberculosis complex demonstrates that Mycobacterium africanum, Mycobacterium bovis, Mycobacterium caprae, Mycobacterium microti and Mycobacterium pinnipedii are later heterotypic synonyms of Mycobacterium tuberculosis. Intern. J. Syst. Evol. Microbiol. 68, 324-332. doi: 10.1099/ijsem.0.002507

Said-Salim, B., Mostowy, S., Kristof, A. S., and Behr, M. A. (2006). Mutations in Mycobacterium tuberculosis Rv0444c, the gene encoding anti-SigK, explain high level expression of MPB70 and MPB83 in Mycobacterium bovis. Mol. Microbiol. 62, 1251-1263. doi: 10.1111/j.1365-2958.2006.05455.x

Scott, C., Cavanaugh, J. S., Pratt, R., Silk, B. J., LoBue, P., and Moonan, P. K. (2016). Human tuberculosis caused by Mycobacterium bovis in the United States, 2006-2013. Clin. Infect. Dis. 63, 594-601.

Shukla, J., Gupta, R., Thakur, K. G., Gokhale, R., and Gopal, B. (2014). Structural basis for the redox sensitivity of the Mycobacterium tuberculosis SigK-RskA sigma-anti-sigma complex. Acta Crystallogr. Sect. D Biol. Crystallogr. 70(Pt 4), 1026-1036. doi: 10.1107/S1399004714000121

Sklar, J. G., Makinoshima, H., Schneider, J. S., and Glickman, M. S. M. (2010). tuberculosis intramembrane protease Rip 1 controls transcription through three anti-sigma factor substrates. Mol. Microbiol. 77, 605-617. doi: 10.1111/j.13652958.2010.07232.x

Staron, A., Sofia, H. J., Dietrich, S., Ulrich, L. E., Liesegang, H., and Mascher, T. (2009). The third pillar of bacterial signal transduction: classification of the extracytoplasmic function (ECF) sigma factor protein family. Mol. Microbiol. 74, 557-581. doi: 10.1111/j.1365-2958.2009.06870.x

Stiefel, A., Mahren, S., Ochs, M., Schindler, P. T., Enz, S., and Braun, V. (2001). Control of the ferric citrate transport system of Escherichia coli: mutations in region 2.1 of the FecI extracytoplasmic-function sigma factor suppress mutations in the FecR transmembrane regulatory protein. J. Bacteriol. 183, 162-170. doi: 10.1128/jb.183.1.162-170.2001

Supply, P., Marceau, M., Mangenot, S., Roche, D., Rouanet, C., Khanna, V., et al. (2013). Genomic analysis of smooth tubercle bacilli provides insights into ancestry and pathoadaptation of Mycobacterium tuberculosis. Nat. Genet. 45, 172-179. doi: $10.1038 / \mathrm{ng} .2517$

Torres-Gonzalez, P., Cervera-Hernandez, M. E., Martinez-Gamboa, A., GarciaGarcia, L., Cruz-Hervert, L. P., Bobadilla-Del Valle, M., et al. (2016). Human tuberculosis caused by Mycobacterium bovis: a retrospective comparison with Mycobacterium tuberculosis in a Mexican tertiary care centre, 2000-2015. BMC Infect. Dis. 16:657. doi: 10.1186/s12879-016-2001-5

van Ingen, J., Rahim, Z., Mulder, A., Boeree, M. J., Simeone, R., Brosch, R., et al. (2014). Characterization of Mycobacterium orygis as M. tuberculosis complex subspecies. Emerg. Infect. Dis. 18, 653-655.

Veyrier, F., Said-Salim, B., and Behr, M. A. (2008). Evolution of the mycobacterial SigK regulon. J. Bacteriol. 190, 1891-1899. doi: 10.1128/jb.01452-07

Conflict of Interest: The authors declare that the research was conducted in the absence of any commercial or financial relationships that could be construed as a potential conflict of interest.

Copyright (C) 2020 Veyrier, Nieves, Lefrancois, Trigui, Vincent and Behr. This is an open-access article distributed under the terms of the Creative Commons Attribution License (CC BY). The use, distribution or reproduction in other forums is permitted, provided the original author(s) and the copyright owner(s) are credited and that the original publication in this journal is cited, in accordance with accepted academic practice. No use, distribution or reproduction is permitted which does not comply with these terms. 